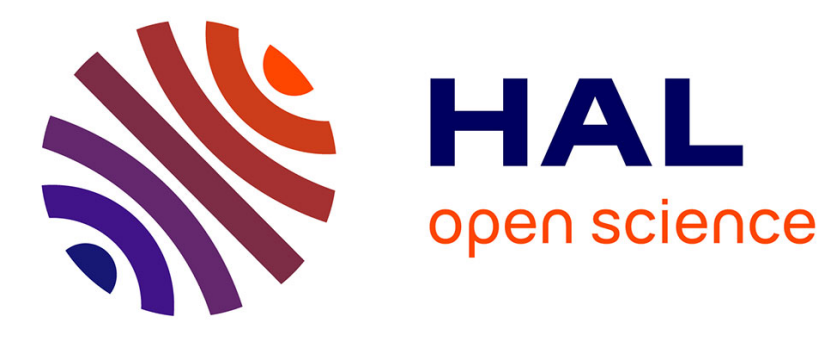

\title{
The MetaCoq Project
}

Matthieu Sozeau, Abhishek Anand, Simon Boulier, Cyril Cohen, Yannick Forster, Fabian Kunze, Gregory Malecha, Nicolas Tabareau, Théo Winterhalter

\section{To cite this version:}

Matthieu Sozeau, Abhishek Anand, Simon Boulier, Cyril Cohen, Yannick Forster, et al.. The MetaCoq Project. Journal of Automated Reasoning, 2020, 10.1007/s10817-019-09540-0 . hal-02167423

\section{HAL Id: hal-02167423 https://hal.inria.fr/hal-02167423}

Submitted on 27 Jun 2019

HAL is a multi-disciplinary open access archive for the deposit and dissemination of scientific research documents, whether they are published or not. The documents may come from teaching and research institutions in France or abroad, or from public or private research centers.
L'archive ouverte pluridisciplinaire HAL, est destinée au dépôt et à la diffusion de documents scientifiques de niveau recherche, publiés ou non, émanant des établissements d'enseignement et de recherche français ou étrangers, des laboratoires publics ou privés. 


\title{
The MetaCoq Project
}

\author{
Matthieu Sozeau, Abhishek Anand, Simon \\ Boulier, Cyril Cohen, Yannick Forster, Fabian \\ Kunze, Gregory Malecha, Nicolas Tabareau \\ and Théo Winterhalter
}

Received: date / Accepted: date

\begin{abstract}
The MetaCoq project ${ }^{1}$ aims to provide a certified meta-programming environment in CoQ. It builds on TEMPLATE-COQ, a plugin for COQ originally implemented by Malecha (2014), which provided a reifier for CoQ terms and global declarations, as represented in the CoQ kernel, as well as a denotation command. Recently, it was used in the CertiCoq certified compiler project (Anand et al., 2017), as its front-end language, to derive parametricity properties (Anand and Morrisett, 2018). However, the syntax lacked semantics, be it typing semantics or operational semantics, which should reflect, as formal specifications in CoQ, the semantics of CoQ's type theory itself. The tool was also rather bare bones, providing only rudimentary quoting and unquoting commands. We generalize it to handle the entire Polymorphic Calculus of Cumulative Inductive Constructions (pCUIC), as implemented by CoQ, including the kernel's declaration structures for definitions and inductives, and implement a monad for general manipulation of CoQ's logical environment. We demonstrate how this setup allows CoQ users to define many kinds of general purpose plugins, whose correctness can be readily proved in the system itself, and that can be run efficiently after extraction. We give a few examples of implemented plugins, including a parametricity translation and a certifying extraction to call-by-value $\lambda$-calculus. We also advocate the use of MetaCoq as a foundation for higher-level tools.
\end{abstract}

M. Sozeau

Pi.R2 Project-Team, Inria Paris and IRIF, France

S. Boulier, N. Tabareau, T. Winterhalter

Gallinette Project-Team, Inria Nantes, France

C. Cohen

Université Côte d'Azur, Inria, France

Y. Forster, F. Kunze

Saarland University, Germany

A. Anand, G. Malecha

BedRock Systems, USA

1 https://metacoq.github.io/metacoq 


\section{Introduction}

Meta-programming is the art of writing programs (in a meta-language) that produce or manipulate programs (written in an object language). In the setting of dependent type theory, the expressivity of the language allows the case were the meta and object languages are actually the same, accounting for well-typedness. This idea has been pursued in the work on inductive-recursive (IR) and quotient inductive-inductive types (QIIT) in Agda to reflect a syntactic model of a dependently-typed language within another one (Chapman, 2009; Altenkirch and Kaposi, 2016). These term encodings include type-correcteness internally by considering only well-typed terms of the syntax, i.e. derivations. However, the use of IR or QIITs complicates considerably the metatheory of the meta-language which makes it difficult to coincide with the object language represented by an inductive type. More problematically in practice, the unification of the syntax and its well-typedness makes it very difficult to use because any function from the syntax can be built only at the price of a proof that it respects typing, conversion or any other features described by the intrinsically typed syntax right away.

Other works have taken advantage of the power of dependent types to do metaprogramming in a more progressive manner, by first defining the syntax of terms and types; and then defining out of it the notions of reduction, conversion and typing derivation (Devriese and Piessens, 2013; Van der Walt and Swierstra, 2013) (the introduction of (Devriese and Piessens, 2013) provides a comprehensive review of related work in this area). This can be seen as a type-theoretic version of the functional programming language designs such as Template HASKell (Sheard and Jones, 2002a) or MetaML (Taha and Sheard, 1997). This is also the approach taken by Malecha in his thesis (Malecha, 2014) where he introduced TEmPLATE-CoQ, a plugin which defines a correspondence - using quoting and unquoting functions-between CoQ kernel terms and inhabitants of an inductive type representing internally the syntax of the calculus of inductive constructions (CIC), as implemented in CoQ. It becomes thus possible to define programs in CoQ that manipulate the representation of CoQ terms and reify them as functions on CoQ terms. Recently, its use was extended for the needs of the CertiCoq certified compiler project (Anand et al., 2017), which uses it as its front-end language. It was also used by Anand and Morrisett (2018) to formalize a modified parametricity translation, and to extract CoQ terms to a CBV $\lambda$-calculus (Forster and Kunze, 2016). All of these translations however lacked any means to talk about the semantics of the reified programs, only syntax was provided by TEMPLATE-CoQ. This is an issue for CertiCoq for example where both a non-deterministic small step semantics and a deterministic call-by-value big step semantics for CIC terms had to be defined and preserved by the compiler, without an "official" specification to refer to.

The MetaCoq project described in this paper remedies this situation by providing a formal semantics of CoQ's type theory, that can independently be refined and studied. The advantage of having a very concrete untyped description of CoQ terms (as opposed to IR or QIITs definitions) together with an explicit type checker is that the extracted type-checking algorithm gives rise to an OCAML program that can directly be used to type-check CoQ kernel terms. This opens a way to a concrete solution to bootstrap Coq by implementing the Coq kernel in Coq. However, a complete reification of CIC terms and a definition of the checker are not enough to provide a meta-programming framework in which CoQ plugins could be implemented. One needs access to CoQ logical environments. We achieve this using the TemplateMonad, which reifies CoQ general commands, such as lookups and declarations of constants and inductive types. 
As far as we know this is the only reflection framework in a dependently-typed language allowing such manipulations of terms and datatypes, thanks to the relatively concise representation of terms and inductive families in CIC. Compared to the MTAC project (Ziliani et al., 2015), LEAN's tactic monad (Ebner et al., 2017), or AGDA's reflection framework (Van der Walt and Swierstra, 2013), our ultimate goal is not to interface with CoQ's unification and type-checking algorithms, but to provide a self-hosted, bootstrappable and verifiable implementation of these algorithms. This opens the possibility to verify the kernel's implementation, a problem tackled by Barras (1999) using set-theoretic models. In addition, we advocate for the use of METACoQ as a foundation to build higher-level tools. For example, translations, boilerplate generators, domain-specific proof languages, or even general purpose tactic languages.

Terminologically, we reserve the use of the name Template-CoQ to denote reification of the internal syntax and logical environment of CoQ, and also for the reification of the type-checking algorithm. We otherwise use the name MetaCoQ when talking about definition of the formal semantics and certification of the algorithms.

Outline of the paper. In Section 2, we present the complete reification of CoQ terms, covering the entire CIC and present a formal specification of typing derivations of these terms. In Section 3, we show the definition of the TemplateMonad for general manipulation of CoQ's logical environment and use it to define plugins for various translations from Coq to CoQ or $\lambda$-calculus (Section 4). Section 5 covers a modification to TemplateMonad that enables plugins to be run natively in OCAML. Finally, we discuss related and future work in Section 6 .

\section{A Formal Specification of CoQ}

In this section, we give a formal specification for Coq by giving syntax and semantics. We will proceed as follows. First, we give the syntax of Coq terms (Section 2.1) and environments (Section 2.2):

term : Set context : Set

Then, we give the formal semantics of those terms by defining the typing relation (Section 2.3), the reduction relation and the conversion relation (Section 2.4):

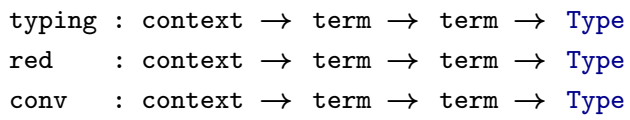

Finally, Section 2.5 is devoted to typing environment and inductive types while Section 2.6 explains the management of universes.

\subsection{Reification of Terms}

The central piece of MetaCoQ is the inductive type term (Figure 1) which represents the syntax of CoQ terms (this language is called Gallina). This inductive follows directly the constr datatype of $\mathrm{COQ}$ terms in the implementation of CoQ, except 


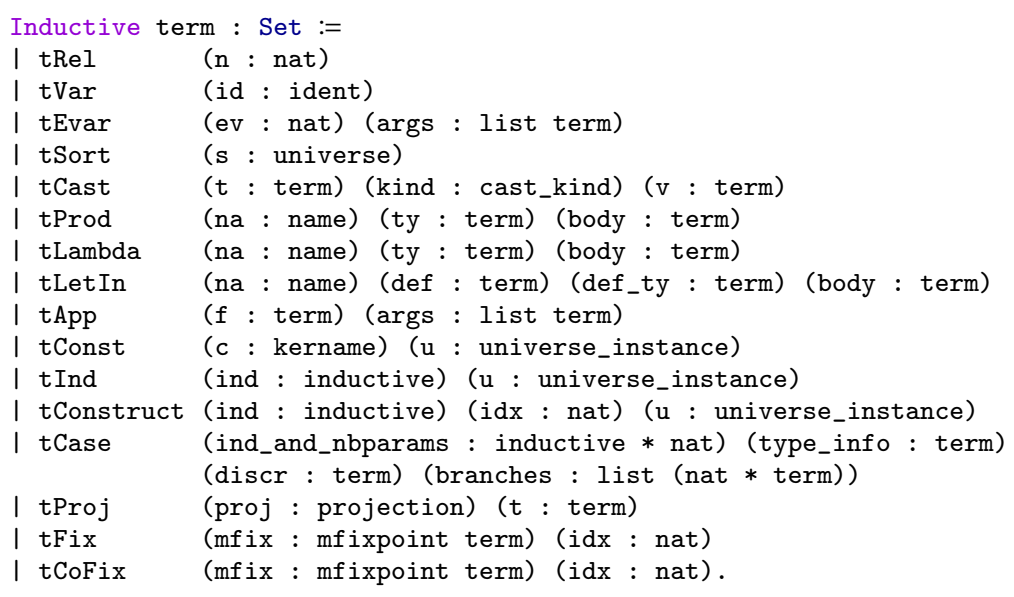

Fig. 1 MetaCoQ's representation of CoQ terms mirrors CoQ's constr type.

for the use of OCAML's native arrays and strings ${ }^{2}$. Some familiar constructions are recognizable: sorts, lambdas, applications, ... Let's review the different constructors.

Constructor tRel represents variables bound by abstractions (introduced by tLambda), dependent products (introduced by tProd) and local definitions (introduced by tLetIn). The natural number is a de Bruijn index. The name is a printing annotation:

Definition ident := string

Inductive name := nAnon | nNamed (_ : ident).

Sorts are represented with tSort, which takes a universe as argument. A universe can be either Prop, Set or a more complex expression representing one of the Type universes. The details are given in Section 2.6.

Type casts ( $t: A$ ) are given by tCast.

$n$-ary application is introduced by tApp. In tApp $\mathrm{t} 1, \mathrm{t}$ is expected not to be an application, and 1 to be a non-empty list.

Example 1 The function fun ( $\mathrm{f}:$ Set $\rightarrow$ Set) (A : Set) $\Rightarrow \mathrm{f} A$ is represented by:

tLambda (nNamed "f")

(tProd nAnon (tSort [(Level.1Set, false)]) (tSort [(Level.1Set, false)]))

(tLambda (nNamed "A") (tSort [(Level.1Set, false)]) (tApp (tRel 1) [tRel 0]))

The three constructors tConst, tInd and tConstruct represent references to constants declared in a global environment. The first is for definitions or axioms, the second for inductive types, and the last for constructors of inductive types. In CoQ, constants can be universe polymorphic, meaning that they can be used at different universe levels. In such a case, said universe levels are given in the universe_instance which is a list of levels. If the constant is not universe polymorphic, the instance is expected to be empty.

tCase represents pattern-matchings, tProj primitive projections, tFix fixpoints and tCoFix cofixpoints.

${ }^{2}$ An upcoming extension of CoQ (Armand et al., 2010) with such features could address this mismatch. 
Example 2 The addition on natural numbers

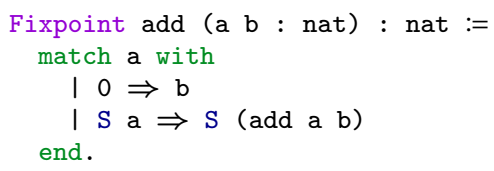

Is represented by:

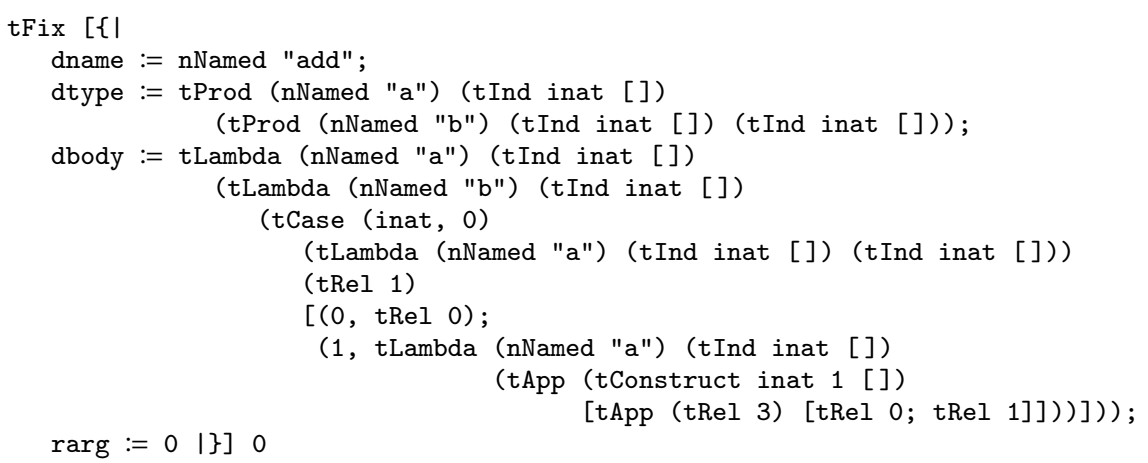

where inat is a notation for the inductive representing nat:

$\{\mid$ inductive_mind := "Coq.Init.Datatypes.nat"; inductive_ind $:=0 \mid\}$

tVar is for named variables introduced in Coq sections or during interactive proofs. tEvar represents for existential variables, i.e. holes to be filled in terms. Typing of these two constructions is not defined in MetaCoq for the moment.

\subsection{Reification of environment}

In Coq, the meaning of a term is relative to an environment, which must be reified as well. We distinguish the global environment which is constant through a typing derivation, from the local context which may vary. The type of typing relation is:

$$
\text { typing : global_context } \rightarrow \text { context } \rightarrow \text { term } \rightarrow \text { term } \rightarrow \text { Type }
$$

(similar for red and conv)

The local context records the types and potential bodies (for let-ins) of de Bruijn indexes:

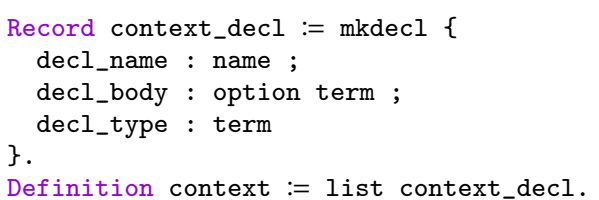

The de Bruijn index 0 is bound to the head of the list. Contexts are written in snoc order: we use the notation $\Gamma$, , d for adding d to the head of $\Gamma$. We also use the abbreviations vass $\mathrm{x}$ A and vdef $\mathrm{x} \mathrm{t}$ A for the two ways to build a context_decl (with or without a body). Last, we use the notation $\Gamma,,, \Gamma^{\prime}$ for context concatenation.

Remark 1 Contrarily to MetaCoq, in the OCAml code of Coq de Bruijn indices start at 1 for historical reasons. 
The global environment consists of two parts: the graph of universes (described in Section 2.6) and a list of declarations, properly ordered according to dependencies.

Definition global_declarations := list global_decl

Definition global_context $:=$ global_declarations $*$ uGraph.t.

A declaration is either the declaration of a constant (a definition or an axiom, according to the presence of body) or of a block of mutual inductive types (which brings both the inductive types and their constructors to the context).

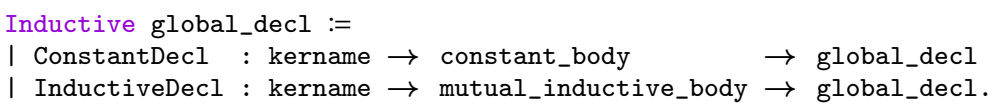

The kernel name kername is a fully qualified name (among modules), for instance the kernel name corresponding to nat is Coq. Init.Datatypes.nat. kername as a type is a synonym to string.

The declaration of a constant is fairly easy:

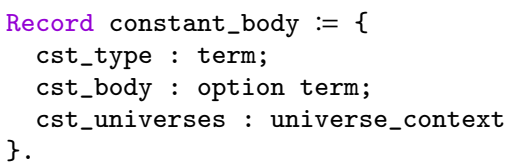

The universe_context indicates whether the constant is polymorphic or not. If so, it contains the constraints that the universe instances have to satisfy.

Declarations of inductives are more involved, they are described in Section 2.5.

\subsection{Typing judgements}

Now that we have terms and environments, we can describe formally all the typing rules of CoQ. This is done by defining an inductive family typing whose definition looks like:

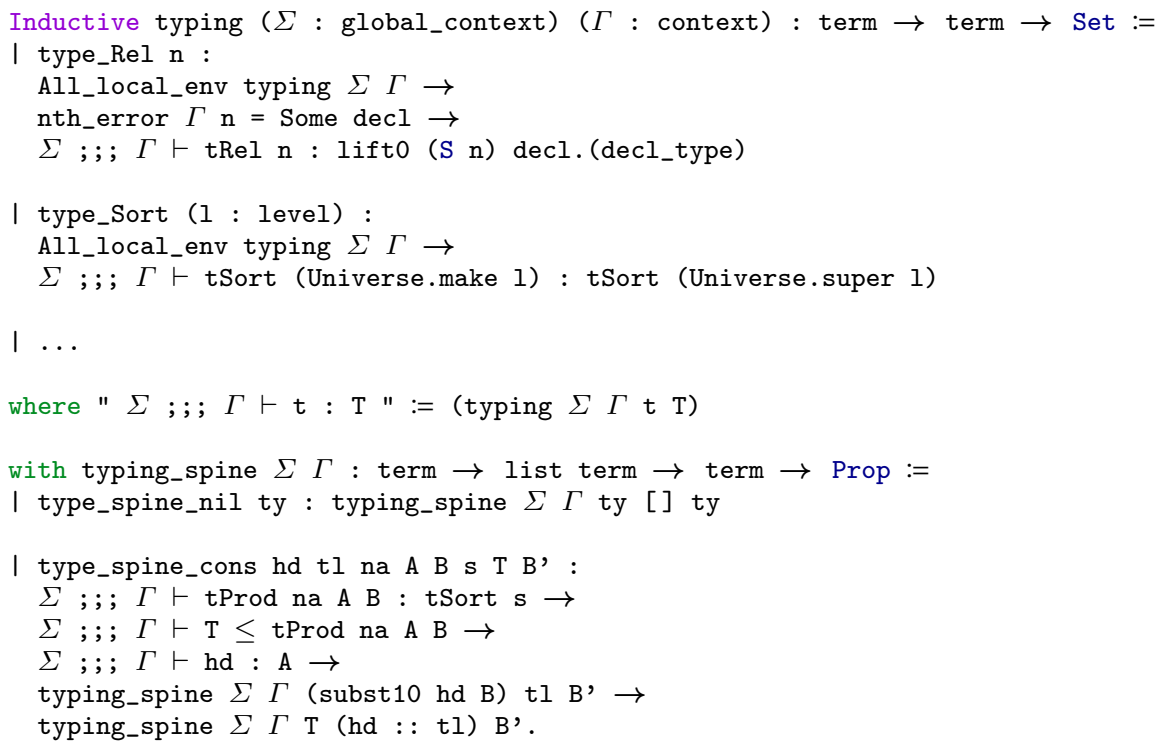


The typing rules include the basic dependent $\lambda$-calculus with let-bindings, global references to inductives and constants, pattern-maching, primitive projections and (co)fixed-points. Universe polymorphic definitions and the well-formedness judgment for global declarations are dealt with as well. The only ingredients missing are the termination check for fixed-points and productivity check for cofixed-points. They are work-in-progress.

Note that the typing rules use substitution and lifting operations of de Bruijn indexes (lifto, subst, ...), their definitions are standard. The typing relation also relies on the subtyping relation. It is described in Section 2.4.

We shall now take time to explain in details the rules one by one.

Variables. A variable is well typed when its de Bruijn index corresponds to a declaration in the (local) context $\Gamma$. The following rule is not saying much more despite its looks.

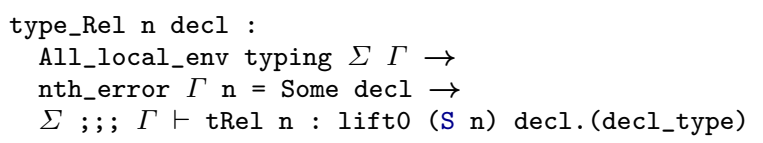

decl is a declaration of type context_decl. The rule attests that the nth variable corresponds to the nth most recent declaration in the context and thus has the ascribed type. The latter is however lifted because the context contains $\mathrm{n}$ declarations after it:

$\Gamma=\Delta, \operatorname{decl}_{-} \mathrm{n}, \ldots, \operatorname{decl}_{1}, \operatorname{dec}_{0}$

with decl_n typed in $\Delta$, so $\Gamma$ is $\Delta$ extended with $\mathrm{S} \mathrm{n}$ declarations, hence the lifto (S $\mathrm{n}$ ). Finally, All_local_env typing $\Sigma \Gamma$ is asserting that the local context $\Gamma$ is well-formed in global context $\Sigma$. Later on this property is called wf_local $\Sigma \Gamma$ but here the dependency on typing is being made explicit.

Sorts. Any sort corresponding to a level (without a +1 ) can be typed with its successor universe (with $\mathrm{a}+1$ ), provided the context is well-formed.

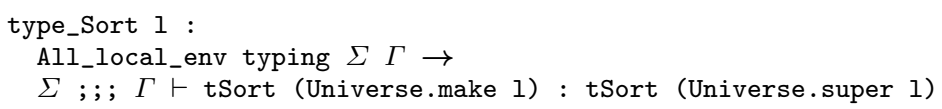

Remark 2 With this rule, only non-algebraic universes can be typed (see Section 2.6 for the definition of non-algebraic universes).

Type-casts. In CoQ, a type-cast happens when you give a type explicitly to an expression: $(t: A) . t$ is checked to have type A and the whole expression is also typed with A.

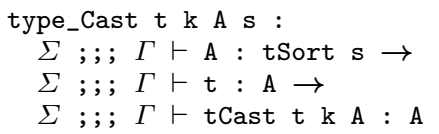

In the rule it is required that A is well-sorted, meaning that there exists (constructively) a sort s such that $\mathrm{A}$ is of type tSort $\mathrm{s}$. In CoQ's kernel, the $\mathrm{k}$ : cast_kind indicates which algorithm is used to check the conversion between A and the type of $t$. We ignore it for the moment in MetaCoq. 
Dependent products. The dependent product, or $\Pi$-type, $\forall \mathrm{x}: \mathrm{A}, \mathrm{B}$ is well typed when both $\mathrm{A}$ and $\mathrm{B}$ are well typed (the latter in the context extended with assumption $\mathrm{x}: \mathrm{A})$.

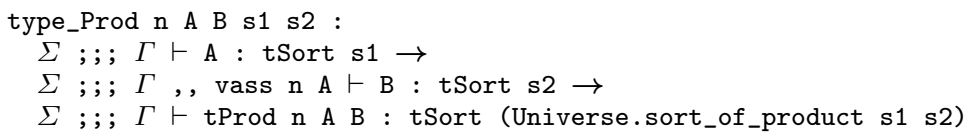

The sort in which the product lives in roughly the maximum of the sorts of its components, accounting for impredicativity of Prop:

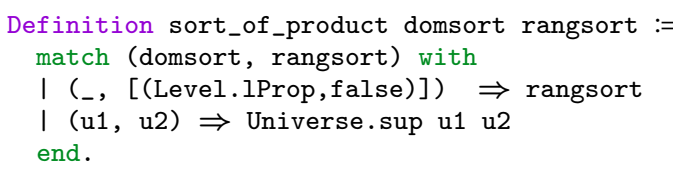

$\lambda$-abstractions. Similarly the rule governing the typing of fun $x: A \Rightarrow t$ is not surprising.

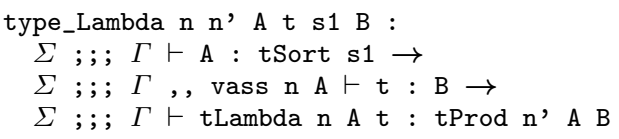

Notice the names $\mathrm{n}$ and $\mathrm{n}$ ' that are different in the term and the type; they are only printing hints and are irrelevant to typing, which is why this rule doesn't force them to be the same.

let in expression. tLetIn $\mathrm{x} b \mathrm{~B} t$ reifies let $\mathrm{x}:=\mathrm{b}: \mathrm{B}$ in $\mathrm{t}$ for which typing is pretty straightforward. Assuming $\mathrm{t}: \mathrm{A}$ the whole expression has type let $\mathrm{x}:=\mathrm{b}: \mathrm{B}$ in $\mathrm{A}$.

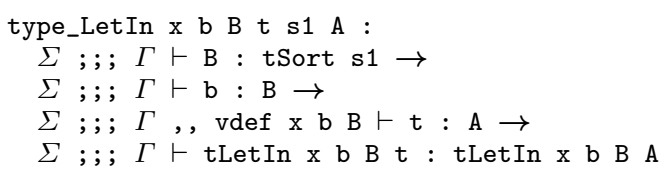

Applications. Typing applications is usually simple, but because MetaCoq features $n$-ary applications, we need to be careful when handling them.

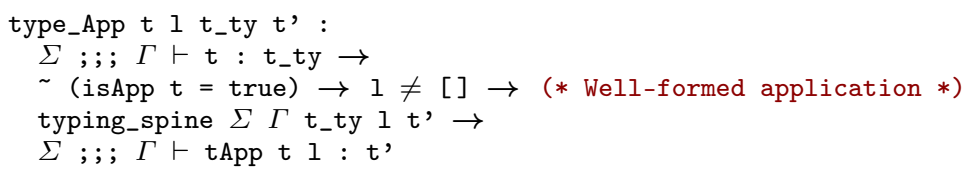

The conditions $\sim$ (isApp $t=$ true) and $1 \neq[]$ ensure that the application is wellformed: that is $t$ is not a nested application and it is applied to at least one argument. Then typing_spine $\Sigma \Gamma$ t_ty 1 t' states that a term of type t_ty applied to a list of arguments 1 will return a term of type t'. Let's have a closer look at it: 


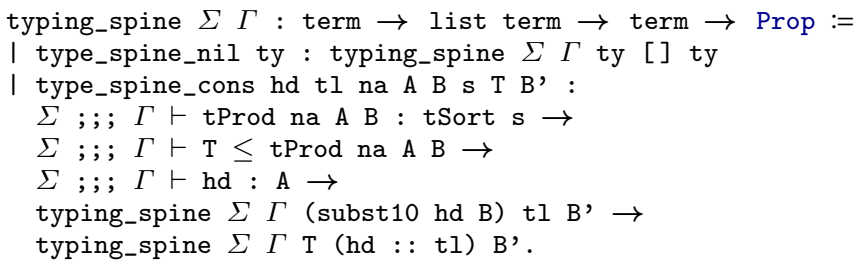

Basically, it iterates over every argument of the function, checking each time that the new function has a function type and is being applied to something in its domain. The argument is then substituted in the codomain which then is matched against a function type again, until there are no arguments left and the type can be returned as is.

Global constants. A constant can either refer to a global definition (stemming from Definition or Lemma for instance), or to an axiom (Axiom). It has a name which is a kername. Such a declaration can be universe polymorphic, so when referring to a constant, one needs to provide it with a universe instance (i.e. values for the universe variables in the definition).

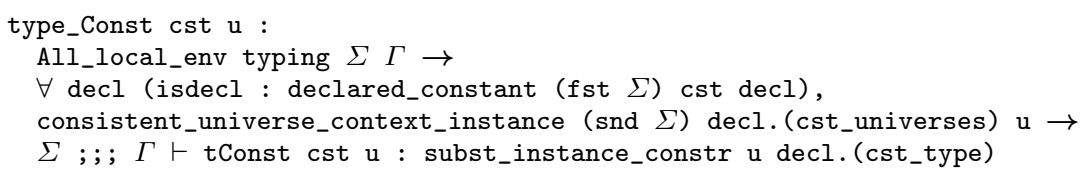

For a constant to be well typed, it first needs to indeed refer to a declared constant in the global context $\Sigma$, which is checked by declared_constant (fst $\Sigma$ ) cst decl, a synonym to lookup_env (fst $\Sigma$ ) cst $=$ Some $($ ConstantDecl cst decl).

consistent_universe_context_instance has a self-explanatory name: it checks that the instance is indeed an instance and verifies that if satisfies the constraints. The constant can thus be typed with the type found in the context decl. (cst_type), where the universes are substituted with the instance.

Inductive types. Typing an inductive type is very similar to typing a constant. This time ind is of type inductive which consists of a kername (the name of the mutualinductive block) and a natural number (the index of the considered inductive type in the block, starting at 0). Similarly to constants, inductive types can be universe polymorphic.

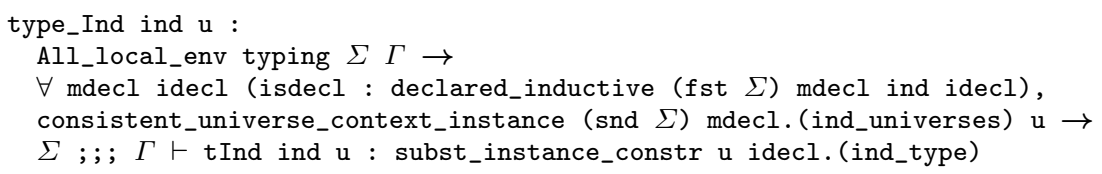

Inductives are declared in the global context as well. mdecl corresponds to the mutual block and idecl corresponds to the inductive of that block we're interested in. declared_inductive checks that ind indeed corresponds to these declarations in $\Sigma$.

Constructors of an inductive type. Inductive types come with their constructors. If the inductive type is declared, and the constructor is indeed a constructor, then it is welltyped. 


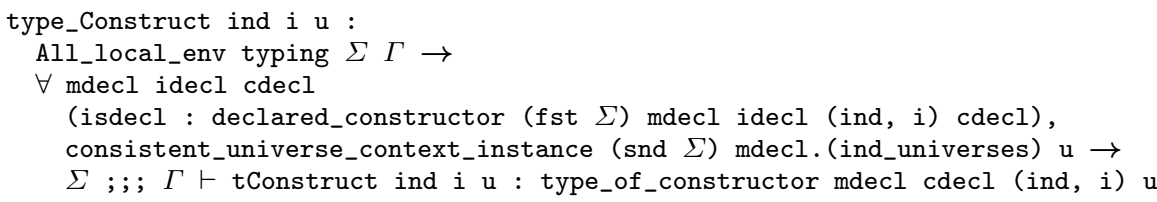

However, this time the constructor types come under the context corresponding to the mutual inductive types. Take for instance the mutual inductive types even and odd:

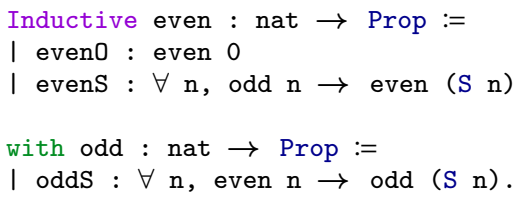

In this case, evens is typed in context even : nat $\rightarrow$ Prop, odd : nat $\rightarrow$ Prop, which is why it can refer to both types, even before they are defined.

The purpose of type_of_constructor is thus to substitute these variables by their actual definitions, as well as instantiating the universes.

Pattern matching. In the internals of Coq and MetaCoq, pattern-matching is refered to as tCase. Dependent pattern-matching with general inductive types is no small task so we shall try and break down the typing rule, and the tCase constructor.

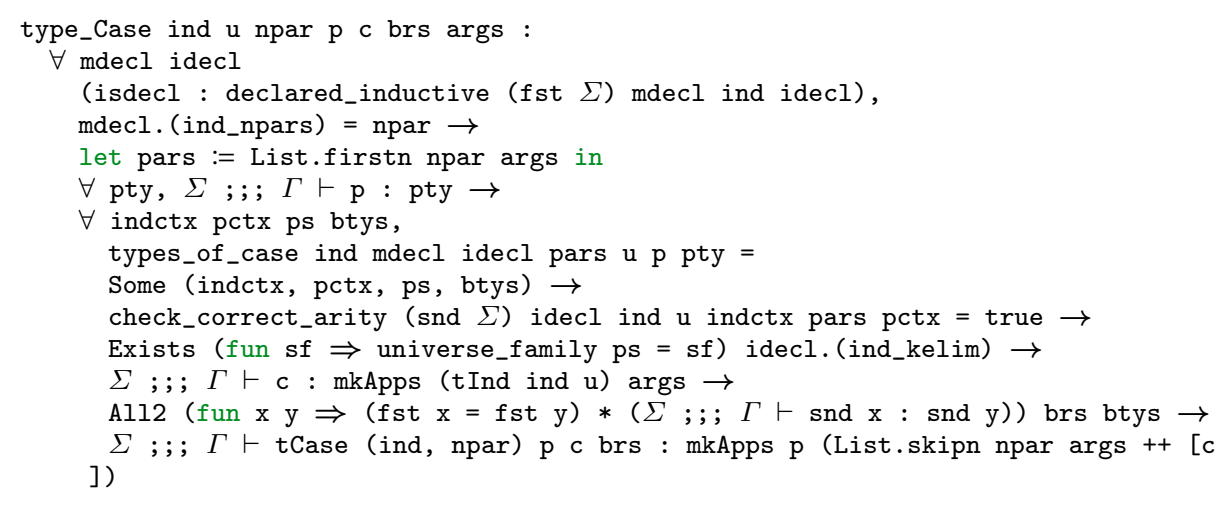

In tCase (ind, npar) $\mathrm{p} \mathrm{c} \mathrm{brs,} \mathrm{ind} \mathrm{is} \mathrm{inductive} \mathrm{type} \mathrm{of} \mathrm{the} \mathrm{scrutinee} \mathrm{c}$, npar is the number of parameters of the inductive (arguments that are constant across all the constructors), $p$ is the predicate or return type, while brs is a list of branches comprised of the number of arguments of the constructor and the term corresponding to the branch (with abstractions for the arguments of the constructor). For instance, consider the following pattern-matching:

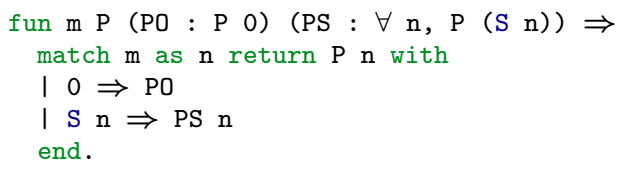

Ignoring the $\lambda \mathrm{s}$, it is quoted to

tCase

(inat, 0) 


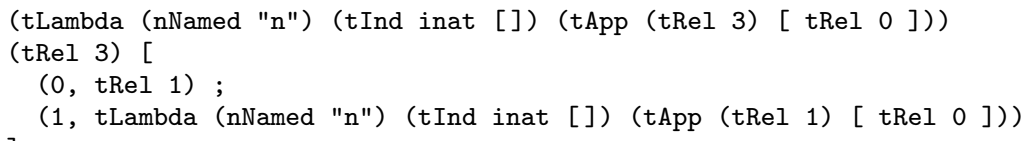

Let's focus on the rule now. As we did for inductive types, we check that the inductive type of the scrutinee is declared.

$\Sigma ; ; ; \Gamma \vdash$ c : mkApps (tInd ind u) args checks that the scrutinee c is indeed in the right type, i.e., the inductive applied to some arguments. After checking that npar is indeed the number of parameters of the inductive type (mdecl. (ind_npars) = npar), we take them off the list of arguments (pars := List.firstn npar args). The rest are the indices of the inductive type and may vary depending on the branch.

Additionally, we check that the predicate (or return type) is well typed with $\Sigma$;; $\Gamma \vdash \mathrm{p}:$ pty.

types_of_case has the purpose of producing the typing information required to type the branches:

- indctx corresponds to the context of the inductive type where the parameters have been instantiated by pars, it thus contains only the indices, (e.g. y : A when matching against $\mathrm{p}$ : @eq $\mathrm{A} \mathrm{u} \mathrm{v,} \mathrm{A} \mathrm{and} \mathrm{u} \mathrm{being} \mathrm{the} \mathrm{parameters);}$

- pctx is the same but for the type of the predicate $\mathrm{p}$ (in the example above it would just be $n$ : nat);

- ps is the sort targeted by $\mathrm{p}$ (basically p quantifies over pctx to return ps - in particular it forces $\mathrm{p}$ to be a type once fully applied);

- btys is a list containing the expected type for each element of brs, the branches.

check_correct_arity verifies that pctx is equal (modulo $\alpha$-renaming) to indctx extended with a variable of the inductive applied to the parameters pars and the variables of context indctx.

Then, Exists (fun sf $\Rightarrow$ universe_family ps $=s f$ ) idecl. (ind_kelim) attests that the sort of the predcate ps belongs to one the universe families that the inductive type can be eliminated to (ind_kelim). The universe family may be Prop, Set or Type and some inductives have restrictions for elimination; most inductive types defined in Prop can only be eliminated into Prop itself, the only to bypass this restriction is using the so-called singleton elimination.

Finally, with All2 we iterate over both brs and btys to check that the branches are indeed typed according to what is recorded in btys, all the while checking that they agree on the number of arguments of the constructors (with the fst part).

Primitive projections. In COQ there are two notions of record types. By default, when one defines the following record:

Record $\mathrm{T}:=\mathrm{mk}\left\{\mathrm{pi}_{1}:\right.$ bool $; \mathrm{pi}_{2}$ : nat $\}$.

it is actually equivalent to the inductive type with one constructor

Inductive $\mathrm{T}:=\mathrm{mk}\left(\mathrm{pi}_{1}: \mathrm{bool}\right)$ ( $\mathrm{pi}_{2}$ : nat).

along with the definitions of $\mathrm{pi}_{1}$ and $\mathrm{pi}_{2}$ by pattern-matching.

It however possible to define records in a more primitive way. Using the global option Set Primitive Projections, the former record definition is still internally represented as an inductive, but this time, additionally to constructors, it has projections, corresponding to $\mathrm{pi}_{1}$ and $\mathrm{pi}_{2}$. Projections can be called with the syntax $\mathrm{t} .\left(\mathrm{p} \mathrm{i}_{1}\right.$ ) or as regular functions. 


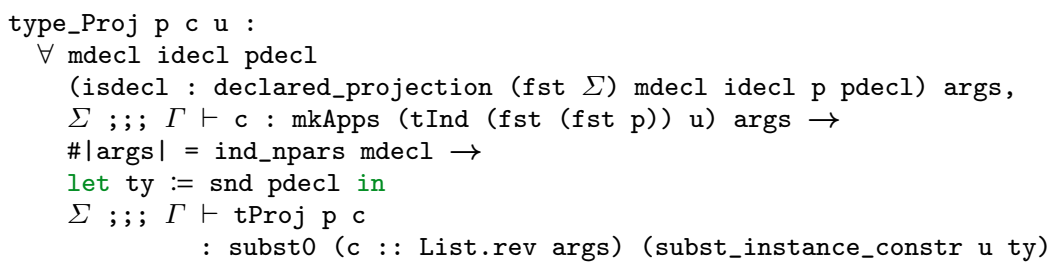

As usual, declared_projection checks that $\Sigma$ contains both the inductive and the projection declaration. The projection is applied to a term $\mathrm{c}$ of the record as ensured by the condition:

$\Sigma ; ; ; \Gamma \vdash \mathrm{c}: \operatorname{mkApps}($ tInd (fst (fst p)) u) args

Here projection stands for inductive * nat $*$ nat, that is an inductive, a number of parameters and the index of the projected argument. We verify that the inductive is fully applied with \#|args| = ind_npars mdecl, stating that the number of arguments corresponds to the number of parameters of the inductive type. Finally, we substitute these arguments, c, and the universes in the type of the projection to get the type of the term.

Fixed-points. In CoQ, the fixed-point operator is primitive and completes patternmatching for performing induction. One usually writes a fixed-point using the aptly named command Fixpoint. It is however possible to write them directly in a term with fix. Let's consider the following mutual fixed-point:

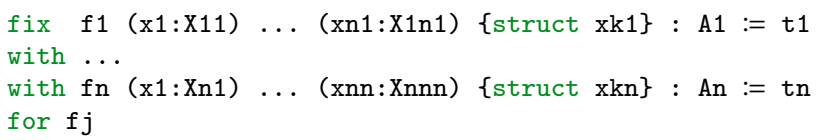

This fixed-point will be of type $\forall\left(x_{1}: X_{j} 1\right) \ldots(x n j: X j n j), A j$. For it to be well typed there are three conditions:

- Each Ai has to be a type;

- Each ti has to be of type Ai in a context extended by the signatures of the fixedpoints (allowing the recursive calls in the body):

$$
\Gamma, f_{1}: A_{1}, \ldots f_{n}: A_{n}, x_{1}: X_{i 1}, \ldots x_{n_{i}}: X_{i n_{i}} \vdash t_{i}: A_{i} ;
$$

- A termination criterion has to be fulfilled. Such a criterion has not yet been implemented in MetaCoq.

Internally, a fixed-point is represented with tFix mfix idx where mfix : list (def term) represents the mutual fixed-points, and idx : nat specifies which of them we want to refer to. def is the following record:

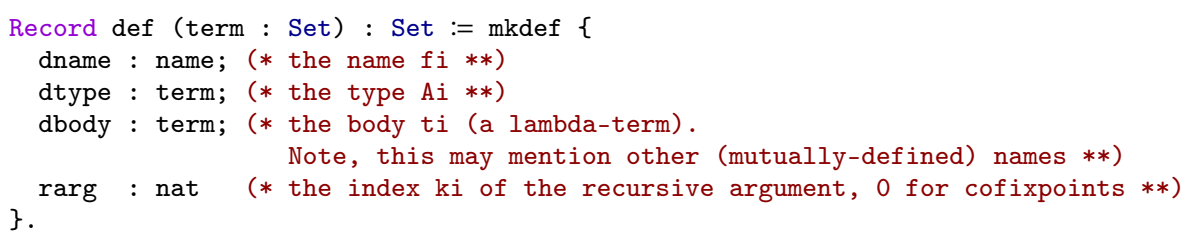

The formal typing rule is the following: 


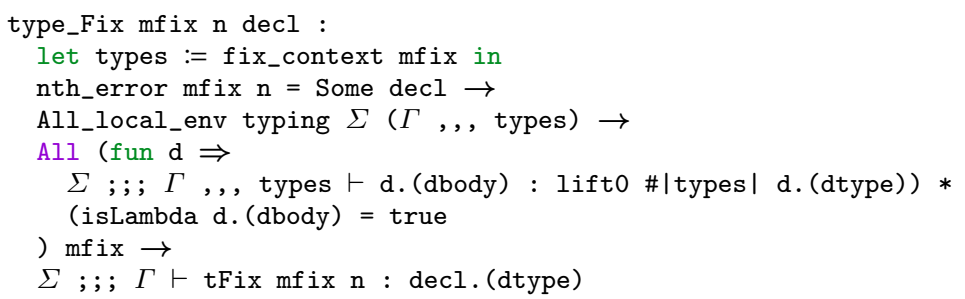

First, we build a context containing the assumptions of the different definitions with types := fix_context mfix, and verify that the composite context $\Gamma$, , types is wellformed. Then we check that idx indeed corresponds to one of the definitions of the block (nth_error $\operatorname{mfix~} \mathrm{n}=$ Some decl). Finally, for each of the definitions, we check that the body has the ascribed type (in the extended context, hence the lifto) and that they all correspond to functions. The return type is the ascribed type.

Cofixed-points. Co-fixed-points are handled in a very similar fashion to regular fixed-points. Even their representation is the same. Again, productivity conditions remain unchecked for the time being.

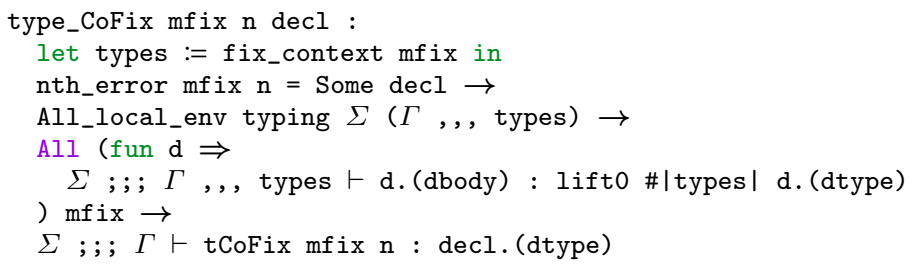

Conversion rules. We conclude with the usual conversion rule.

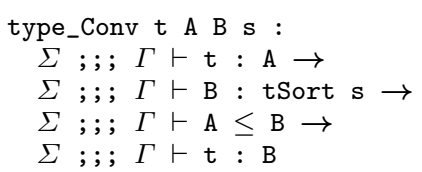

It is here stated with cumulativity (allowing to increase universes in contravariant positions), and it requires the new type to be well-sorted as well. We shall explain conversion and cumulativity in more details in the next subsection.

\subsection{Conversion, Cumulativity and Reduction}

The cumulativity, or subtyping, relation, is defined from one-step reduction red1 as follows:

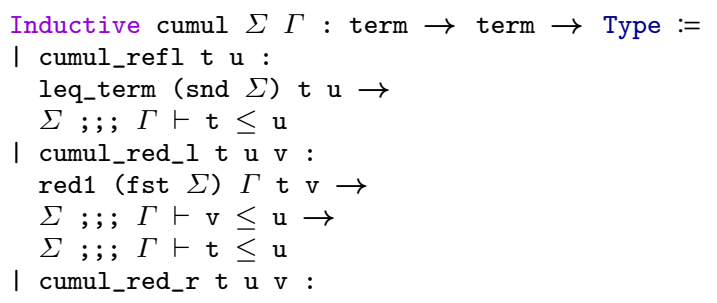




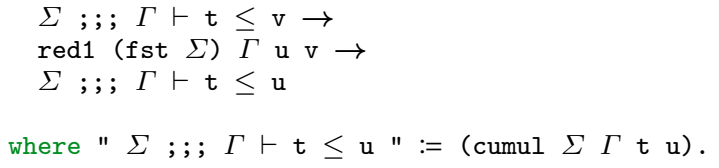

Basically, $\mathrm{A} \leq \mathrm{B}$ when $\mathrm{A}$ and $\mathrm{B}$ respectively reduce to A' and B' such that cumulativity can be checked syntactically with leq_term. leq_term operates as a congruence and invokes universe comparison when reaching sorts.

Conversion is derived from cumulativity going both ways:

Definition conv $\Sigma \Gamma \mathrm{T} \mathrm{U}:=$

$(\Sigma ; ; \quad \Gamma \vdash \mathrm{T} \leq \mathrm{U}) *(\Sigma ; ; \quad \Gamma \vdash \mathrm{U} \leq \mathrm{T})$.

Notation " $\Sigma ; ; ; \Gamma \vdash \mathrm{t}=\mathrm{u} ":=(\operatorname{conv} \Sigma \Gamma \mathrm{t} \mathrm{u})$.

It is equivalent to having both terms reduce to $\alpha$-convertible terms.

The main point of interest is thus how one-step reduction red1 is defined. It is introduced with the following command:

Inductive red1 ( $\Sigma$ : global_declarations) $(\Gamma:$ context $):$ term $\rightarrow$ term $\rightarrow$ Type

however, we will not put here all of its constructors. Most of them are congruence rules. For instance, for tLambda, the congruences are as follows.

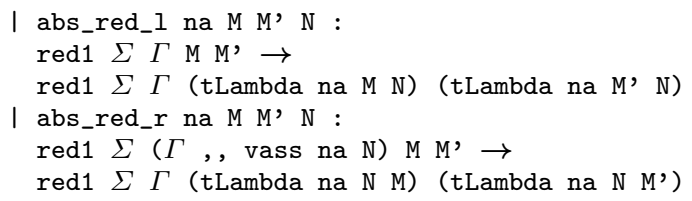

A term reduces to another in one step, if one of its subterms does. It holds for all term constructors so we will now focus on actual computation rules.

$\beta$-reduction. A $\lambda$-abstraction may consume its first argument to reduce.

red_beta na $\mathrm{t}$ b a 1 :

red1 $\Sigma \Gamma$ (tApp (tLambda na t b) (a : : 1)) (mkApps (subst10 a b) 1)

let expressions. A let expression can be unfolded as a substitution right away (this is called $\zeta$-reduction):

red_zeta na b t b' :

red1 $\Sigma \Gamma($ tLetIn na b t b') (subst10 b b')

It can also be unfolded later, by reducing a reference to the let-binding:

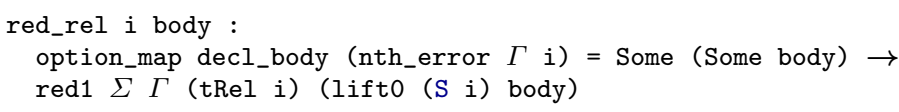

It checks that the ith variable in $\Gamma$ corresponds to a definition and replaces the variable with it. It needs to be lifted because the body was defined in a smaller context. 
Pattern-matching. A match expression can be reduced with $\iota$-reduction when the scrutinee is a constructor.

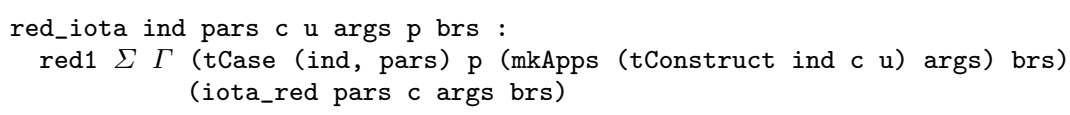

Herein, iota_red is defined as follows:

Definition iota_red npar c args brs :=

mkApps (snd (List.nth c brs (0, tDummy))) (List.skipn npar args).

As List.nth takes a default value, (0, tDummy) can be ignored, it basically picks the branch corresponding to the constructor and applies it to the indices of the inductive (List.skipn npar args).

Fixed-point unfolding. Even after they are checked to be terminating, fixed-points cannot be unfolded indefinitely. There is a syntactic guard to only unfold a fixed-point when its recursive argument is a constructor.

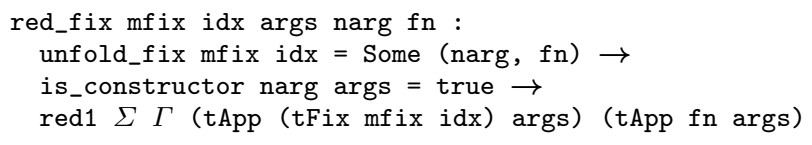

unfold_fix mfix idx allows to recover both the body (fn) and the index of the recursive argument (narg) while is_constructor narg args checks that the said argument is indeed a constructor.

Co-fixed-point unfolding. There are two cases where a co-fixed-point gets unfolded. One of them is when it is matched against.

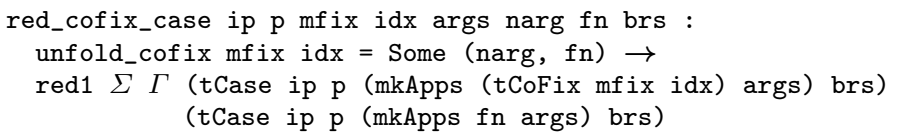

As for fixed-points, unfold_cofix returns the body.

A co-fixed-point can also be unfolded when projected, behaving exactly the same way.

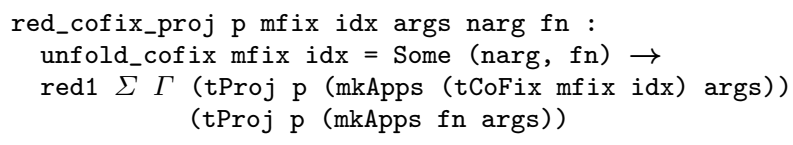

$\delta$-reduction. $\delta$-reduction allows to unfold a constant (from the global context $\Sigma$ ).

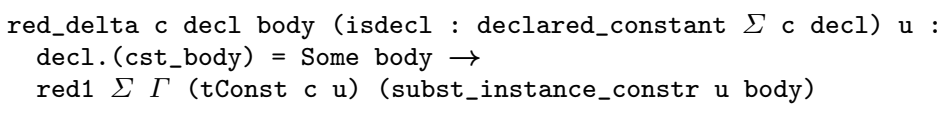

It can only be done if a definition is indeed found. Its universes (if it is universe polymorphic) are then instantiated. 
Projection. When a constructor of a record is projected, it can be reduced to the corresponding field.

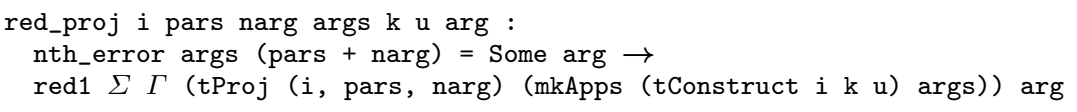

2.5 Typing environments

Local environment. As already mentionned in the typing rules, a local context $\Gamma$ is wellformed if wf_local $\Sigma \Gamma$ holds. This type is an abbreviation of All_local_env typing $\Sigma \Gamma$ where All_local_env is defined by:

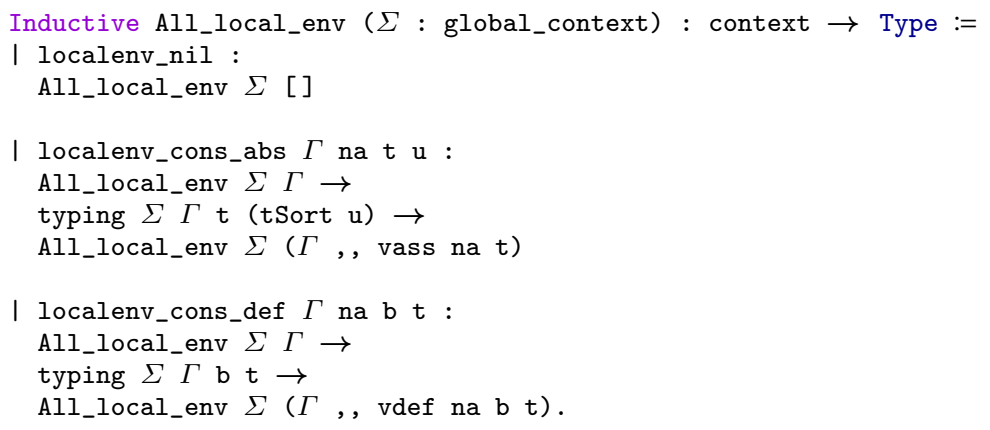

Hence, the empty context is well-formed. A variable assumption is well-formed if the type is well-sorted and a variable definition is well-formed if the body is indeed of the given type.

The well-typedness of the local context is enforced in every typing judgment:

typing_wf_local: $\forall \Sigma \Gamma \mathrm{t}$ T, wf $\Sigma \rightarrow \Sigma ; ; \quad \Gamma \vdash \mathrm{t}: \mathrm{T} \rightarrow$ wf_local $\Sigma \Gamma$

Global environment. As opposed to local contexts, the well-typedness of the global environment is not enforced in typing judgments and have thus to be stated additionally with the predicate wf $\Sigma$ (as above for instance). This predicate is defined as on_global_decls (fst $\Sigma$ ) (snd $\Sigma$ ) where we have:

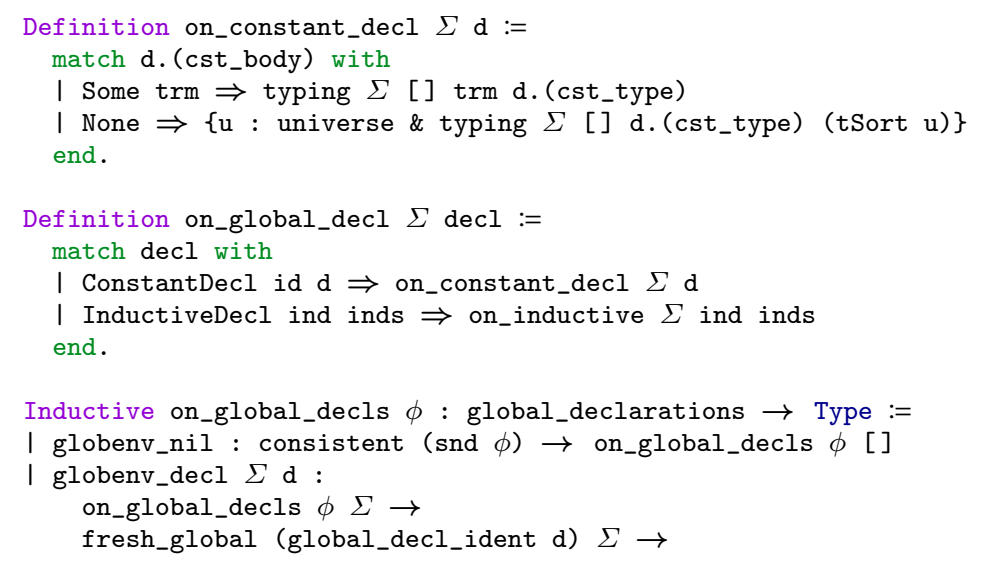


on_global_decl $(\Sigma, \phi) \mathrm{d} \rightarrow$
on_global_decls $\phi(\mathrm{d}:: \Sigma)$.

The empty environment is wellformed when the graph of universes has no inconsistencies. Well-formedness of constants is the same as for local contexts. Well-formedness of inductive declarations is outlined below. For each new declaration, the identifier is required to be fresh with respect to the previous ones.

Inductive declarations. In CoQ, a block of mutual inductive types is declared as follows:

$\begin{array}{ll}\text { Inductive } & \text { I1 params : } \mathrm{A} 1:=\mathrm{c} 11: \mathrm{T} 11|\ldots| \mathrm{c} 1 \mathrm{n} 1: \mathrm{T} 1 \mathrm{n} 1 \\ \ldots & \text { Ip params : } \mathrm{Ap}:=\mathrm{cp} 1: \mathrm{Tp} 1|\ldots| \mathrm{cpnp}: \text { Tpnp. } \\ \text { with }\end{array}$

I1, . I Ip are the names of the inductive types. A1, . Ap are the arities. The cij are the constructors and the Tij their types. params is the context of parameters. This context can contain some let-bindings, we will write $x_{1}, \ldots x_{n}$ for the variables without body bound in this context.

Remark 3 With respect to indices, parameters $x_{1}, \ldots x_{n}$ have to be constant in all the conclusions of the types of constructors. However, they may vary in the types of arguments of constructors. A parameter is called uniform if it is constant through the whole inductive type, and non uniform otherwise.

In MetaCoq, a mutual block of inductive types is formally represented by a mutual_inductive_body which, itself, consists mainly in a list of one_inductive_body, one for each block.

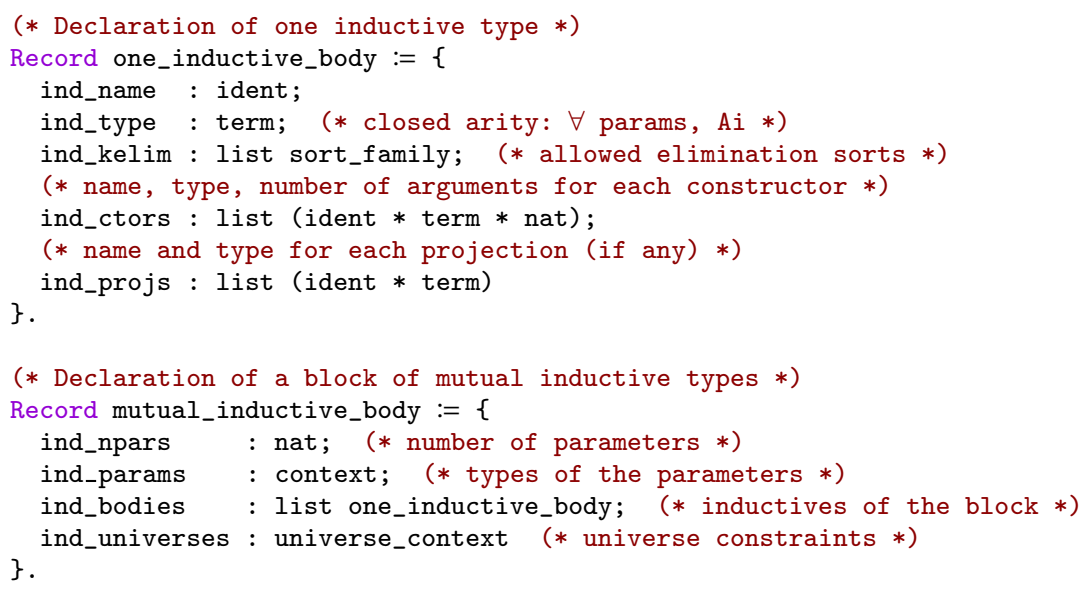

A block mutual_inductive_body is well-formed when:

- the context of parameters is well-formed: wf_local $\Sigma$ ind_params;

- ind_npars is the number of assumptions (i.e. without let-in) in ind_params;

- each one_inductive_body is well-formed.

And a declaration of type one_inductive_body is well-formed when:

- the arity ind_type is well-sorted in the empty context and starts with at least ind_npars foralls " $\forall$ " (skipping the lets and casts); 
- for each triplet (id, $\mathrm{T}, \mathrm{n}$ ) of the list of constructors ind_ctors,

$-\mathrm{T}$ is well-sorted under the context of arities:

$$
I_{1}: A_{1}^{\prime}, \ldots I_{n}: A_{n}^{\prime} \vdash T: s \quad \text { where } A_{i}^{\prime} \text { is } \forall \text { params, } A_{i} \text {; }
$$

- $\mathrm{T}$ is of the shape $\forall$ params args, $I_{i} x_{1} \ldots x_{n} t_{1} \ldots t_{k}$ where args are the real arguments of the constructor and $I_{i}$ is the corresponding de Bruijn index;

- for each pair (id, T) of the list of projections ind_projs:

- the inductive type has no index;

- $\mathrm{T}$ is well-sorted in the context of parameters extended by the considered inductive type:

$$
\text { params, } x: I_{i} x_{1} \ldots x_{n} \vdash T: s .
$$

This specification of inductive types is not fully complete: for instance ind_kelim is not checked yet. The main missing feature is the positivity criterion.

Remark 4 In CoQ internals, there are in fact two ways of representing a declaration: either as a "body" or as an "entry". The kernel takes entries as input, type-checks them and elaborates them into bodies. In MetaCoq, we provide both, as well as an erasing function mind_body_to_entry from bodies to entries for inductive types.

\subsection{Universes}

The treatment of universes in COQ is both a strong feature and something hard to understand. We hope that MetaCoq can shed some light on it.

Coq relies on a hierarchy of universes: Prop, Set, Type ${ }_{0}, \mathrm{Type}_{1}, \mathrm{Type}_{2}, \ldots$ The universe Set can be seen as a strict synonym of Typeo.

The hierarchy behaves as follows for typing:

$$
\begin{aligned}
& \text { Prop: } \text { Type }_{1} \\
& \text { Type }_{0}: \text { Type }_{1}: \text { Type }_{2} \ldots
\end{aligned}
$$

And as follows with respect to cumulativity:

$$
\text { Prop } \subseteq \text { Type }_{0} \subseteq \text { Type }_{1} \subseteq \text { Type }_{2} \ldots
$$

In CoQ, the user does not have to provide the universe level $i$ of Type ${ }_{i}$ but can instead use typical ambiguity and simply write Type. The CoQ system has then the responsibility of instantiating the universe levels properly. For flexibility, the universe levels are not definitely determined at declaration time. Instead, a universe variable for the level is introduced and only the most general constraints on this variable are recorded. In technical cases, the user can enforce the universe variable with the notation Type $\{1\}$.

For instance, the following definition

Definition $\mathrm{T}:$ Type@ $\left\{\mathrm{l}_{1}\right\}:=\forall\left(\mathrm{A}:\right.$ Type@ $\left.\left\{\mathrm{l}_{2}\right\}\right), \mathrm{A} \rightarrow$ Set.

will generate the constraints Set $<l_{1}$ and $l_{2}<l_{1}$ where $l_{1}$ and $l_{2}$ are universe variables. Here, the set of constraints is satisfiable: it can be instantiated with, for instance, $\left(l_{1}:=2, l_{2}:=1\right)$.

The Coq system maintains a set of constraints and updates it each time a new universe variable is introduced. The CoQ system also manipulates some algebraic universes which are of the form Type $\left\{\max \left(l_{1}, l_{2}+1\right)\right\}$, as introduced in Herbelin and Spiwack 
(2013). The level of these universes is uniquely determined by $l_{1}$ and $l_{2}$. Thanks to the Set keyword, Type $e_{0}$ is the only Type ${ }_{i}$ that can be given explicitly by the user.

Formally, a universe is the supremum of a (non-empty) list of level expressions, and a level is either Prop, Set, a global level or a de Bruijn polymorphic level variable. Polymorphic levels are used when type checking a polymorphic declaration (constant or inductive).

Inductive level := 1Prop | 1Set | Level (_ : string) | Var (_: $\mathbb{N}$ ). Definition universe $:=$ list (level $*$ bool). (* level+1 if true $*$ )

A universe is called non-algebraic if it is a level (that is, of the form $[(1, f a l s e)])$, and algebraic otherwise.

A constraint is given by two levels and a constraint_type:

Inductive constraint_type := Lt | Le | Eq.

Definition univ_constraint $:=$ Level.t $*$ constraint_type $*$ Level.t.

The set of constraints (constraints) is implemented by sets as lists without duplicates coming from the CoQ standard library. A valuation is an instance for all monomorphic and polymorphic levels in natural numbers. Monomorphic (global) levels are required to be positive so that we have Prop : Type for any instance.

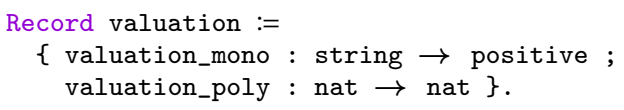

We define the evaluation of valuation on monomorphic levels and then on universes.

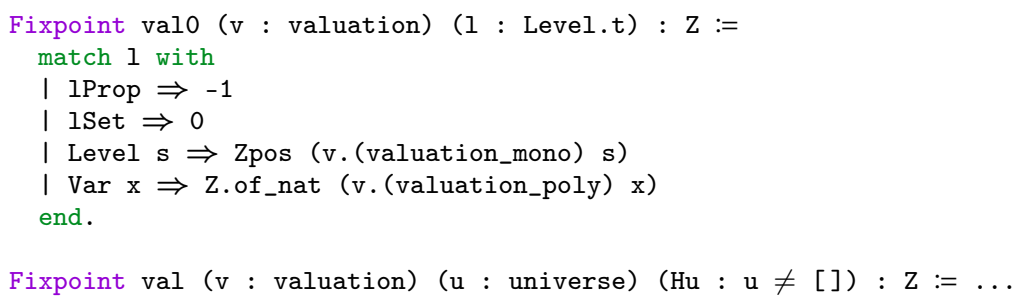

Satisfaction of constraints is defined as expected. Then, a set of constraints is said to be consistent if there exists a valuation satisfying the constraints:

Definition consistent $\operatorname{ctrs}:=\exists \mathrm{v}$, satisfies $\mathrm{v}$ ctrs

Last, given a set of constraints, two universes are said equal when they are equal for all valuation satisfying the constraints (idem for $\leq$ ):

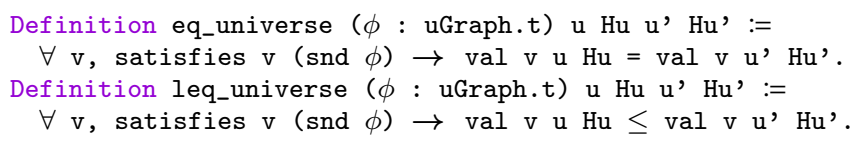

The functions eq_term and leq_term used in conversion and cumulativity relations are defined as congruence on terms calling those two functions on sorts.

\subsection{Toward Coq bootstrap}

The reification of syntax is a first step toward the bootstrap of Coq. From this, one can reimplement some algorithm of the kernel such as type inference, type checking, 
the test of conversion/cumulativity and so on. On the other hand, the reification of semantics is then a first step toward the certification of such reimplementation. From here, we can dream of a proof assistant whose critical algorithms are certified.

As a preliminary stage, we implemented the three aforementioned algorithms:

(* typing_result is an error monad $*$ )

check_conv: Fuel $\rightarrow$ global_ctx $\rightarrow$ context $\rightarrow$ term $\rightarrow$ term $\rightarrow$ typing_result unit

infer $\quad:$ Fuel $\rightarrow$ global_ctx $\rightarrow$ context $\rightarrow$ term $\rightarrow$ typing_result term

check $\quad:$ Fuel $\rightarrow$ global_ctx $\rightarrow$ context $\rightarrow$ term $\rightarrow$ term $\rightarrow$ typing_result unit

Type checking is given by type inference followed by a conversion test. All the rules of type inference are straightforward except for cumulativity. The cumulativity test is implemented by comparing recursively head normal forms for a fast-path failure. We implemented weak-head reduction by mimicking CoQ 's implementation, which is based on an abstract machine inspired by the KAM. CoQ 's machine optionally implements a variant of lazy, memoizing evaluation (the lazy reduction strategy). That feature has not been implemented yet. A major difference with the OCAML implementation is that all of functions are required to be shown terminating in CoQ. One possibility could be to prove the termination of type-checking separately but this requires to prove in particular the normalization of CIC which is a complex task. Instead, we simply add a fuel parameter to make them syntactically recursive and make makeOutofFuel a type error.

We also implemented, the satisfiability check of universe constraints. In CoQ, the set of constraints is maintained as a weighted graph called the universe graph. The nodes are the introduced level variables, and the edges are given by the constraints. Each edge has a weight which corresponds to the minimal distance needed between the two nodes:

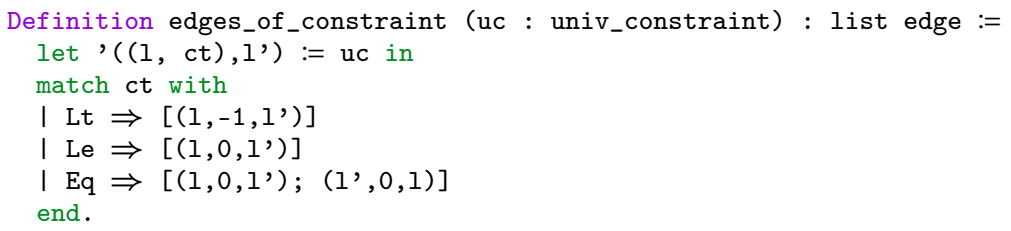

We implemented some functions to manipulate the graph:

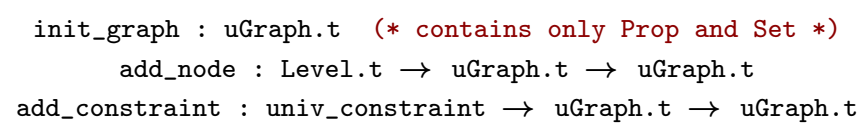

And some functions to query the graph:

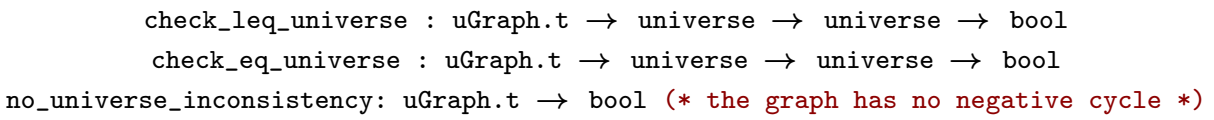

For the moment they all rely on a naive implementation of the Bellman-Ford algorithm as presented in Cormen et al. (2009).

\section{The Template-Coq Plugin}

Along with the formal specification of CoQ, the METACoq project also provides a plugin, called Template-CoQ, which allows to move back and forth from concrete 
syntax (the syntax of CoQ as entered by the user) to reified syntax (as defined in the previous section).

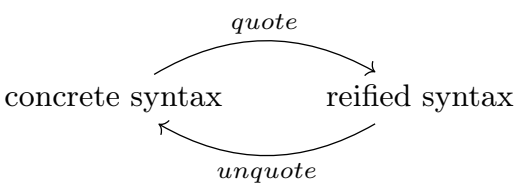

The plugin can reflect all kernel CoQ terms.

We start by presenting the basic commands provided by the plugin to quote and unquote (Section 3.1), and then we describe in Section 3.2 the reification of the main COQ vernacular commands which can be used to automatize the use of quoting and unquoting. This makes it possible in particular to write plugins directly in CoQ by combining such commands.

\subsection{Basic commands}

Quoting and unquoting of terms. The command Test Quote reifies the syntax of a term and prints it. For instance,

Test Quote (fun $\mathrm{x} \Rightarrow \mathrm{x}+0$ ).

outputs the following

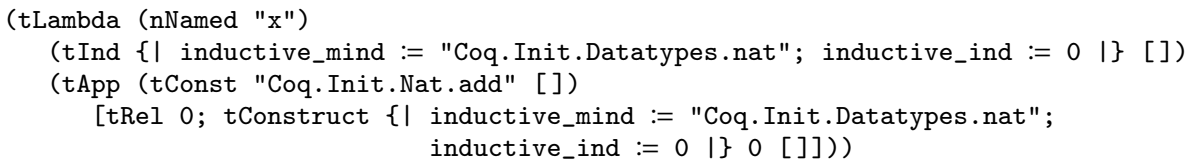

The command Quote Definition $f:=$ ( $f$ un $x \Rightarrow x+0$ ) records the reification of the term in the definition $f$ to allow further manipulations.

On the converse, the command Make Definition constructs a term from its syntax. The example below defines zero to be 0 of type $\mathbb{N}$.

Make Definition zero := tConstruct (mkInd "Coq.Init.Datatypes.nat" 0) 0 [].

where mkInd na $\mathrm{k}:$ inductive is the $\mathrm{k}^{\text {th }}$ inductive of the mutual block of the name na.

Quoting and unquoting the environment. TEMPLATE-CoQ provides the command Quote Recursively Definition to quote an environment. This command crawls the environment and quotes all declarations needed to typecheck a given term.

For instance, the command Quote Recursively Definition mult_syntax := mult (the multiplication on natural numbers) will define mult_syntax of type global_declarations

* term. This first component is the list of declarations needed to typecheck the term mult. Namely, the declaration of the inductive nat and of the constants add and mult. The second component is the reified syntax of the term, here it is only: tConst "Coq. Init.Nat.mult" [].

The command Make Inductive provides a way to declare an inductive type from its syntax. For instance, the following command defines a copy of $\mathbb{N}$ :

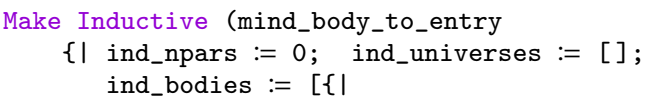




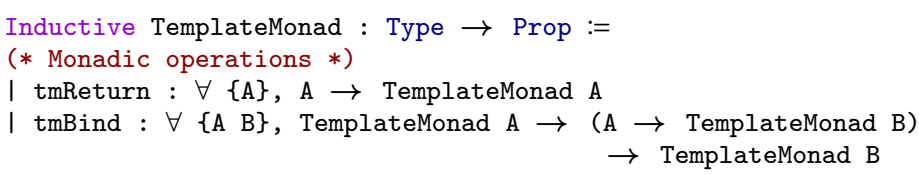

(* General commands *)

I tmPrint : $\forall\{\mathrm{A}\}, \mathrm{A} \rightarrow$ TemplateMonad unit

I tmMsg : string $\rightarrow$ TemplateMonad unit

I tmFail : $\forall\{$ A $\}$, string $\rightarrow$ TemplateMonad A

I tmEval : reductionStrategy $\rightarrow \forall\{\mathrm{A}\}, \mathrm{A} \rightarrow$ TemplateMonad A

I tmDefinition : ident $\rightarrow \forall\{\mathrm{A}\}, \mathrm{A} \rightarrow$ TemplateMonad A

I tmAxiom : ident $\rightarrow \forall$ A, TemplateMonad A

I tmLemma : ident $\rightarrow \forall$ A, TemplateMonad A

I tmFreshName : ident $\rightarrow$ TemplateMonad ident

I tmAbout : qualid $\rightarrow$ TemplateMonad (option global_reference)

I tmCurrentModPath : unit $\rightarrow$ TemplateMonad string

I tmExistingInstance : qualid $\rightarrow$ TemplateMonad unit

I tmInferInstance : option reductionStrategy $\rightarrow \forall \mathrm{A}$, TemplateMonad (option A)

(* Quoting and unquoting commands *)

I tmQuote : $\forall\{\mathrm{A}\}, \mathrm{A} \rightarrow$ TemplateMonad term

I tmQuoteRec : $\forall\{$ A $\}, A \rightarrow$ TemplateMonad (global_declarations $*$ term)

I tmQuoteInductive : qualid $\rightarrow$ TemplateMonad mutual_inductive_body

I tmQuoteUniverses : TemplateMonad uGraph.t

I tmQuoteConstant : qualid $\rightarrow$ bool $\rightarrow$ TemplateMonad constant_entry

I tmMkInductive : mutual_inductive_entry $\rightarrow$ TemplateMonad unit

I tmUnquote : term $\rightarrow$ TemplateMonad $\{\mathrm{A}:$ Type \& A\}

I tmUnquoteTyped : $\forall \mathrm{A}$, term $\rightarrow$ TemplateMonad A.

Fig. 2 The monad of commands

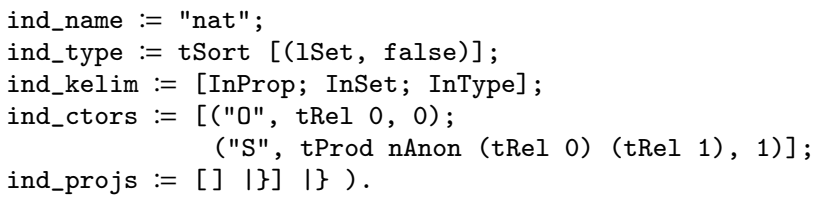

More examples on the use of quoting/unquoting commands can be found in the file test-suite/demo.v.

\subsection{Reification of CoQ Commands}

Along with the reification of Coq terms, Template-Coq provides the reification of the main vernacular commands of CoQ. This way, one can write plugins by combining such commands. To combine commands while taking into account that commands have side effects (notably by interacting with global environment), we use the "free" monadic setting to represent those operations. A similar approach was for instance used in Mtac (Ziliani et al., 2015).

The syntax of reified commands is defined by the inductive family TemplateMonad (Fig. 2). In this family, TemplateMonad A represents a program which will eventually output a term of type A. There are special constructors tmReturn and tmBind to provide 
(freely) the basic monadic operations. We use the monadic syntactic sugar $x \leftarrow t ;$; $u$ for tmBind $t$ ( $f u n ~ x \Rightarrow u$ ) and ret for tmReturn.

The other operations of the monad can be classified in two categories:

- the traditional CoQ operations (tmDefinition to declare a new definition, etc.)

- the quoting and unquoting operations to move between CoQ term and their syntax or to work directly on the syntax (tmMkInductive to declare a new inductive from its syntax for instance).

An overview of available commands is given in Table 1.

\begin{tabular}{|c|c|c|}
\hline $\begin{array}{l}\text { Vernacular } \\
\text { command }\end{array}$ & $\begin{array}{l}\text { Reified command with } \\
\text { its arguments }\end{array}$ & Description \\
\hline Eval & tmEval red $t$ & $\begin{array}{l}\text { Returns the evaluation of } t \text { following the evaluation } \\
\text { strategy red (cbv, cbn, hnf, all, lazy or unfold ) }\end{array}$ \\
\hline Definition & tmDefinition id $t$ & $\begin{array}{l}\text { Makes the definition id := } \mathrm{t} \text { and returns the cre- } \\
\text { ated constant id }\end{array}$ \\
\hline Axiom & tmAxiom id A & $\begin{array}{l}\text { Adds the axiom id of type } A \text { and returns the created } \\
\text { constant id }\end{array}$ \\
\hline Lemma & tmLemma id $\mathrm{A}$ & $\begin{array}{l}\text { Generates an obligation of type A, returns the cre- } \\
\text { ated constant id when all obligations are closed }\end{array}$ \\
\hline \multirow[t]{7}{*}{$\begin{array}{l}\text { About or } \\
\text { Locate }\end{array}$} & tmAbout id & $\begin{array}{l}\text { Returns Some gr if id is a constant in the current } \\
\text { environment and gr is the corresponding global } \\
\text { reference. Returns None otherwise }\end{array}$ \\
\hline & $\begin{array}{l}\text { tmPrint } t \\
\text { tmMsg msg }\end{array}$ & Prints a term or a message \\
\hline & tmFail msg & Fails with error message msg \\
\hline & tmQuote $t$ & Returns the syntax of $t$ (of type term) \\
\hline & tmQuoteRec $t$ & $\begin{array}{l}\text { Returns the syntax of } t \text { and of all the declarations } \\
\text { on which it depends }\end{array}$ \\
\hline & tmQuoteInductive $\mathrm{kn}$ & Returns the declaration of the inductive $\mathrm{kn}$ \\
\hline & $\begin{array}{c}\text { tmQuoteConstant } \mathrm{kn} \\
\mathrm{b}\end{array}$ & $\begin{array}{l}\text { Returns the declaration of the constant } \mathrm{kn} \text {, if } \mathrm{b} \text { is } \\
\text { true the implementation bypass opacity to get the } \\
\text { body of the constant }\end{array}$ \\
\hline \multirow[t]{3}{*}{$\begin{array}{l}\text { Make } \\
\text { Inductive }\end{array}$} & tmMkInductive $\mathrm{d}$ & Declares the inductive denoted by the declaration $\mathrm{d}$ \\
\hline & tmUnquote $\mathrm{tm}$ & $\begin{array}{l}\text { Returns the dependent pair }(A ; t) \text { where } t \text { is the } \\
\text { term whose syntax is tm and A it's type }\end{array}$ \\
\hline & tmUnquoteTyped A tm & $\begin{array}{l}\text { Returns the term whose syntax is tm and checks } \\
\text { that it is indeed of type A }\end{array}$ \\
\hline
\end{tabular}

Table 1 Main Template-Coq commands

A program prog of type TemplateMonad A can be executed with the command Run TemplateProgram prog. This command is thus an interpreter for TemplateMonad programs. It is implemented in OCAML as a traditional CoQ plugin. The term produced by the program is discarded but, and it is the point, a program can have many side effects 
like declaring a new definition, declaring a new inductive type or printing something. Typically, we run programs of type TemplateMonad unit.

Let's look at some examples. The following program adds two definitions foo := 12 and bar $:=$ foo +1 to the current context.

Run TemplateProgram (foo $\leftarrow$ tmDefinition "foo" 12 ;

tmDefinition "bar" (foo +1$))$.

The program below asks the user to provide an inhabitant of nat (here we provide $3 * 3$ ), records it in the lemma foo, prints its normal form, and records the syntax of its normal form in foo_nf_syntax (hence of type term). We use PROGRAM's obligation mechanism ${ }^{3}$ to ask for missing proofs, running the rest of the program when the user finishes providing it. This enables the implementation of interactive plugins.

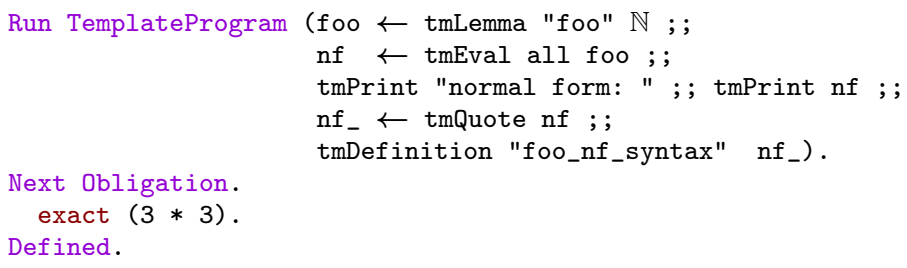

The basic commands of Template-Coq described in 3.1 are implemented with such TemplateProgram. For instance:

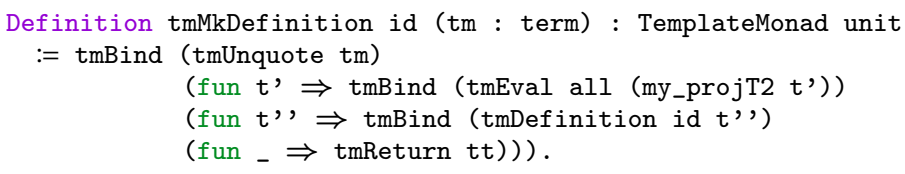

\section{Writing Coq plugins in CoQ}

The reification of commands of CoQ allows users to write CoQ plugins directly inside COQ, without requiring another language like OCAML or an external compilation phase.

In this section, we describe three examples of such plugins: (i) a plugin that adds a constructor to an inductive type, (ii) a plugin for extending CoQ via syntactic translation as advocated in (Boulier et al., 2017) and (iii) a plugin extracting CoQ functions to weak-call-by-value $\lambda$-calculus.

\subsection{A Toy Example: A Plugin to Add a Constructor}

Our first example is a toy example to show the methodology of writing plugins in Template-Coq. Given an inductive type I, we want to declare a new inductive type I' which corresponds to I plus one more constructor.

For instance, let's say that we have a syntax for lambda calculus:

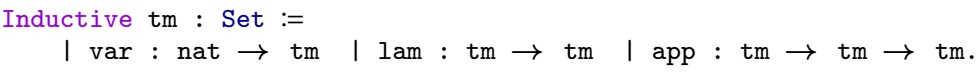

${ }^{3}$ In CoQ, a proof obligation is a goal which has to be solved to complete a definition. Obligations were introduced by Sozeau (2007) in the Program mode. 
And that in some part of our development, we want to consider a variation of tm with a new constructor, e.g. a "let in" constructor. Then we declare tm' with the plugin by:

Run TemplateProgram

(add_constructor tm "letin" (fun $\mathrm{tm}$ ' $\Rightarrow \mathrm{tm}$ ' $\rightarrow \mathrm{tm}$ ' $\rightarrow \mathrm{tm}{ }^{\prime}$ )).

This command has the same effect as declaring the inductive tm' by hand:

Inductive $\mathrm{tm}$ ' : Set :=

| var': nat $\rightarrow$ tm $\quad \mid$ lam, $: \mathrm{tm}^{\prime} \rightarrow \mathrm{tm}$ '

I app' : tm $\rightarrow \mathrm{tm}, \rightarrow \mathrm{tm}$, | letin : $\mathrm{tm}, \rightarrow \mathrm{tm}, \rightarrow \mathrm{tm}$ '.

but with the benefit that if $\mathrm{tm}$ is changed, for instance by annotating the lambda or adding one new constructor, then $\mathrm{tm}^{\prime}$ ' is automatically changed accordingly. We provide other examples, e.g. with mutual inductives, in the file test-suite/add_constructor.v.

We will see that it is fairly easy to define this plugin using Template-Coq. The main function is add_constructor which takes an inductive type ind (whose type is not necessarily Type if it is an inductive family), a name idc for the new constructor and the type ctor of the new constructor, abstracted with respect to the new inductive.

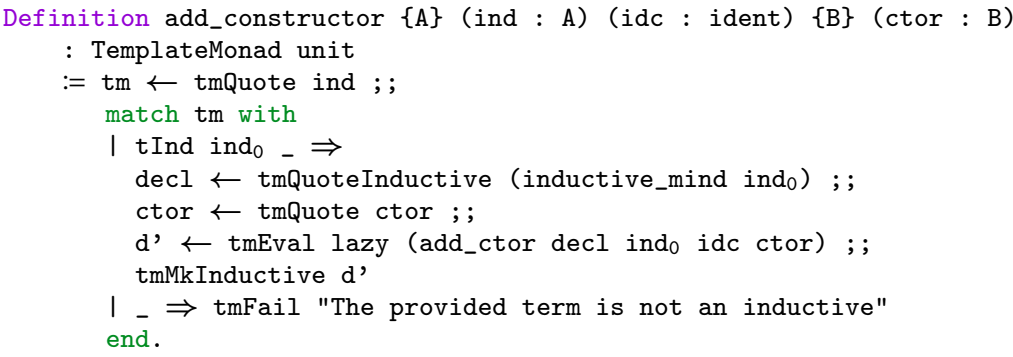

It works in the following way. First the inductive type ind is quoted, the obtained term tm is expected to be a tInd constructor otherwise the function fails. Then the declaration of this inductive is obtained by calling tmQuoteInductive, the constructor is reified too, and an auxiliary function is called to add the constructor to the declaration. After evaluation, the new inductive type is added to the current context with tmMkInductive.

It remains to define the add_ctor auxiliary function to complete the definition of the plugin. It takes a mutual_inductive_body which is the declaration of a block of mutual inductive types and returns another mutual_inductive_body.

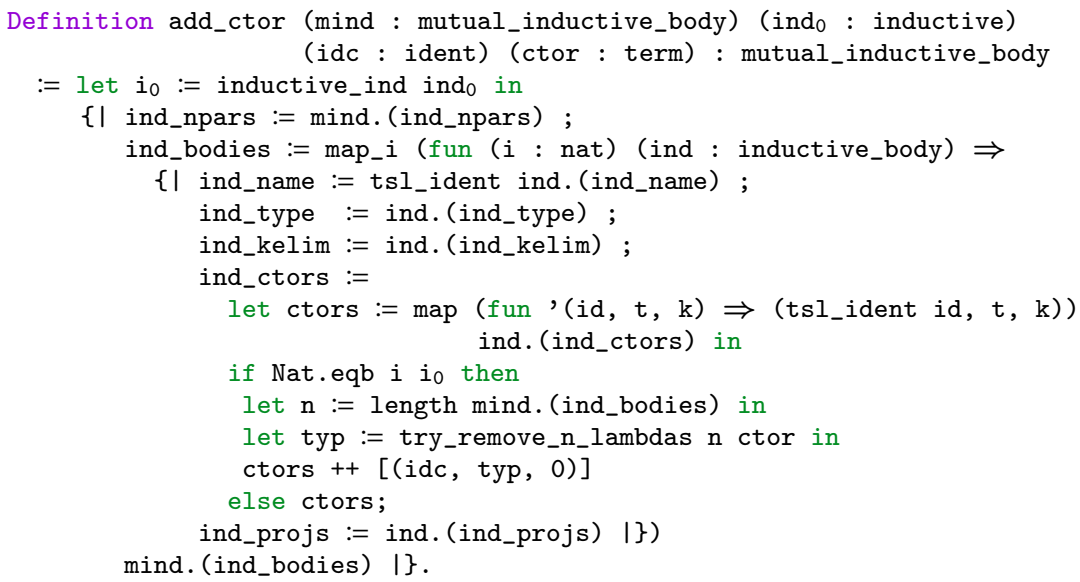


The declaration of the block of mutual inductive types is a record. The field ind_bodies contains the list of declarations of each inductive of the block. We see that most of the fields of the records are propagated, except for the names which are translated to add some primes and ind_ctors, the list of types of constructors, for which, in the case of the relevant inductive ( $i_{0}$ is its number), the new constructor is added.

\subsection{The Program Translations Plugin}

The following plugin expects a syntactic translation as defined in Boulier et al. (2017). It makes it possible to manipulate translated terms and, ultimately, to justify some logical extensions of COQ by postulating safe axioms. It is implemented in the file translations/translation_utils.v.

Two examples of syntactic translations are presented here: the parametricity translation, and a "times bool" translation (which justifies the negation of functional extensionality). A few other examples are available in the directory translations.

In all generality, a translation is given by two functions [ $]_{-}$and $\llbracket-\rrbracket$ from CoQ terms to CoQ terms such that they enjoy at least computational soundness and typing soundness:

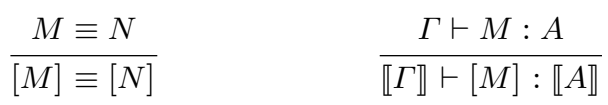

Given such a translation, the plugin provides four commands:

- Translate which computes the translation $[M]$ of a term $M$.

- TranslateRec which computes the translation of a term and of all constants on which it depends.

- Implement. This command computes the translation $\llbracket A x \rrbracket$ of a type Ax and asks the user to inhabit $\llbracket A x \rrbracket$ in proof mode. If the user succeeds (but not before), it declares an axiom of type Ax. If the program translation is sound (cf. Boulier et al. (2017)), it ensures that the axiom does not break consistency.

- ImplementExisting which is used to provide the translation of some terms by hand. It can be used to "implement" an existing axiom. It is also useful to experiment with translations only partially defined; for instance to provide the translation of a particular inductive type without defining the translation of all inductive types.

To work, the plugin needs a translation. It is given by the following record:

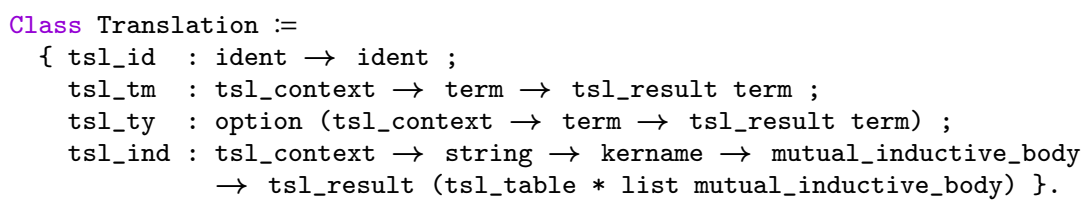

This record is a Class so that, using type classes inference, when a translation is provided, it is automatically found by CoQ.

- tsl_ident is how identifiers are translated. It will always be ( $f$ un id $\Rightarrow$ id ++ "t") for us. 
- tsl_tm is the main translation function implementing [ - ]. It takes a term and returns a term. The translation context contains the global environment and the previously translated constants, see below. The result is in the tsl_result monad which is an error monad:

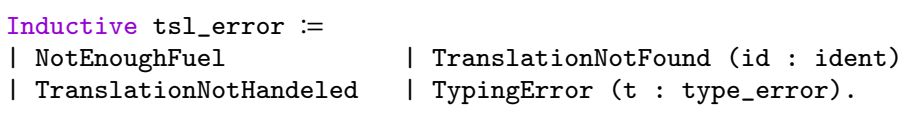

The returned term can be of any type. tsl_tm is used by the commands Translate and TranslateRec.

- tsl_ty is the function translating types $\llbracket$ - $\rrbracket$. This time, the returned term is expected to be a type. This function is used by the commands Implement and ImplementExisting which are not available when tsl_ty is not provided. This is the case for models which do not translate a type by a type (for instance: the standard model, the setoid model, ...).

- Last, tsl_ind is the function translating inductive types. It returns:

- an extended translation table with the translations of the inductive type and its constructor;

- a list of inductive declarations which are used in the translation of the inductive type. Generally, an inductive is translated either by itself (in which case the list is empty), or by a new inductive whose constructors are the the translation of the original constructors (in which case the list is of length one).

The second argument of tsl_ind is technical: it is the path to the module in which the new inductives will be declared.

Translation context. In the translation plugin, the constants (definitions, axioms, inductive types and constructors), are translated one by one. They are recorded in a translation table so that the constants are not retranslated each time they appear. This association table is implemented as the list of the translated constants together with their translation.

Definition tsl_table $:=$ list (global_reference $*$ term).

Thus, the tConst case in the tsl_tm functio is generally implemented by:

I tConst s univs $\Rightarrow$ lookup_tsl_table table (ConstRef s)

and similarly for tInd and tConstruct.

Some translations that we implemented need to access the global environment in which the considered term makes sense. That's why we define a translation context to be a global environment and a translation table:

Definition tsl_context $:=$ global_context $*$ tsl_table.

\subsubsection{Parametricity}

Let's describe the use of the plugin for the parametricity translation. Its implementation can be found in translations/param_original.v.

The translation that we use here follows Reynolds'parametricity (Reynolds, 1983; Wadler, 1989). We follow the already known approaches of parametricity for dependent type theories (Bernardy et al., 2012; Keller and Lasson, 2012). We get an alternative 


$$
\begin{aligned}
{[t]_{0} } & =t \\
{[x]_{1} } & =x^{t} \\
{[\forall(x: A) \cdot B]_{1} } & =\lambda f \cdot \forall\left(x:[A]_{0}\right)\left(x^{t}:[A]_{1} x\right) \cdot[B]_{1}(f x) \\
{[\lambda(x: A) \cdot t]_{1} } & =\lambda\left(x:[A]_{0}\right)\left(x^{t}:[A]_{1} x\right) \cdot[t]_{1} \\
\llbracket \Gamma, x: A \rrbracket & =\llbracket \Gamma \rrbracket, x:[A]_{0}, x^{t}:[A]_{1} x
\end{aligned}
$$

$$
\begin{gathered}
\Gamma \vdash t: A \\
\hline \llbracket \Gamma \rrbracket \vdash[t]_{0}:[A]_{0} \\
\llbracket \Gamma \rrbracket \vdash[t]_{1}:[A]_{1}[t]_{0}
\end{gathered}
$$

Fig. 3 Unary parametricity translation and soundness theorem, excerpt (from Bernardy et al. (2012))

implementation Lasson's plugin PARAMCOQ ${ }^{4}$. For the moment, only the unary case is implemented. The translation is reminded in Figure 3.

The two components of the translation [ - $_{0}$ and $\left[{ }_{-}\right]_{1}$ are implemented by two recursive functions tsl_paramo and tsl_-param ${ }_{1}$.

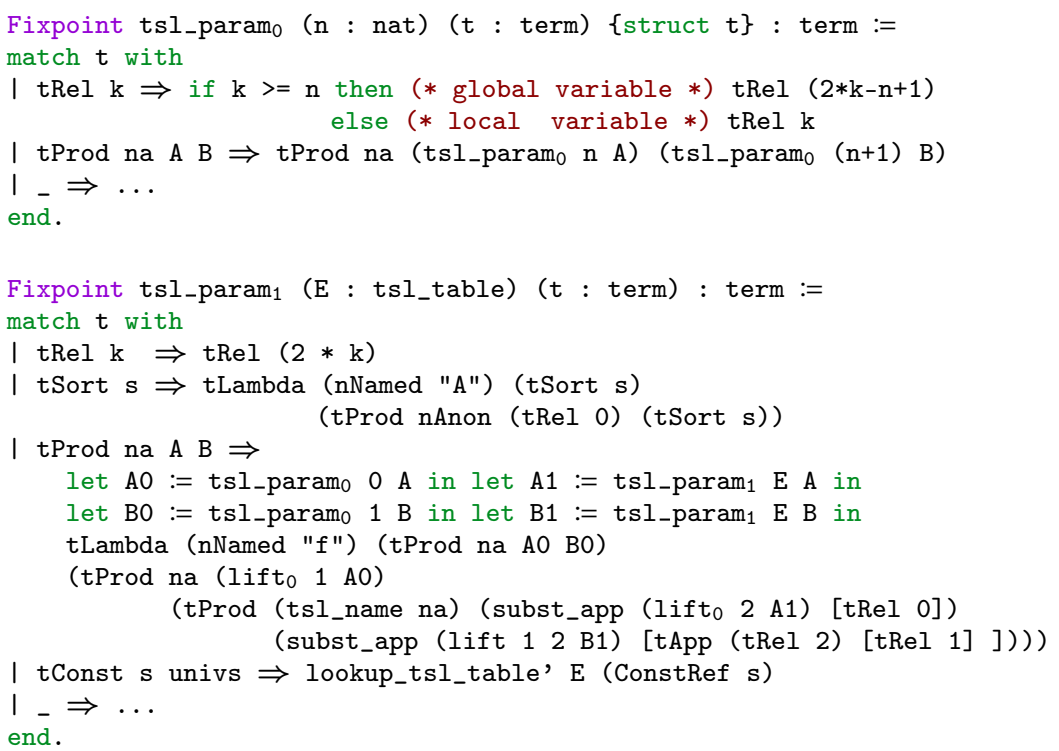

In Figure 3, the translation is presented in a named setting. As a consequence, the introduction of new variables does not change references to existing ones and that's why [- $]_{0}$ is the identity. In the de Bruijn setting of Template-CoQ, the translation has to take into account the shift induced by the duplication of the context. Therefore, the implementation tsl_paramo of [ - $_{0}$ is no longer the identity. The argument $\mathrm{n}$ of tsl_paramo represents the de Bruijn level from which the variables have to be duplicated.

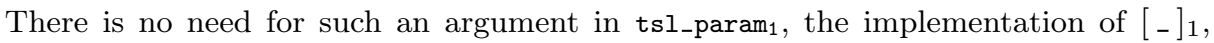
because in this function all variables are duplicated. The implemented cases include pattern matching. Fixed-points are still work in progress.

Given those two functions, we can already translate some terms. For example, the translation of the type of polymorphic identity functions can be obtained by:

Definition ID $:=\forall \mathrm{A}, \mathrm{A} \rightarrow \mathrm{A}$.

\footnotetext{
4 https://github.com/parametricity-coq/paramcoq
} 
Run TemplateProgram (Translate emptyTC "ID").

emptyTC is the empty translation context. This defines ID $^{\mathrm{t}}$ to be:

fun $\mathrm{f}: \forall \mathrm{A}, \mathrm{A} \rightarrow \mathrm{A} \Rightarrow \forall \mathrm{A}\left(\mathrm{A}^{\mathrm{t}}: \mathrm{A} \rightarrow\right.$ Type $)(\mathrm{x}: \mathrm{A}), \mathrm{A}^{\mathrm{t}} \mathrm{x} \rightarrow \mathrm{A}^{\mathrm{t}}(\mathrm{f} \mathrm{A} \mathrm{x})$

We have also implemented tsl_mind_body the translation of inductive types. For instance, the translation of the equality type eq produces the following inductive:

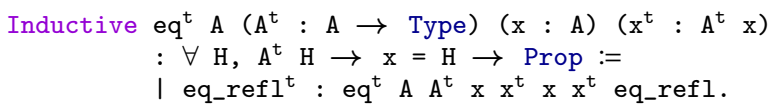

Then $[e q]_{1}$ is given by eq ${ }^{t}$ and [eq_refl $]_{1}$ by eq_ref $l^{t}$.

The translation of the declarations of a block of mutual inductive types are similar declarations, with the arities and the types of constructors translated accordingly.

All put together, the translation is declared by:

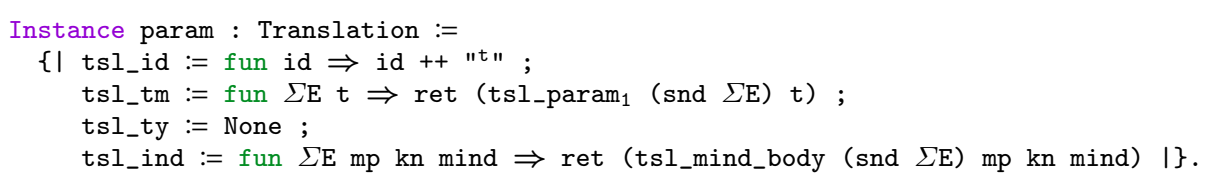

For each constant $c$ of type $A$, it is $[c]_{1}$ (of type $\left.[A]_{1}[c]_{0}\right)$ which is recorded in the translation table. There is no implementation of tsl_ty because there is no meaningful function $\llbracket-\rrbracket$ for this presentation of parametricity.

Example. With this translation, the only commands that can be used are Translate and TranslateRec. Here is an illustration of their use coming from the work of Lasson on the automatic proofs of $\omega$-groupoid laws using parametricity Lasson (2014). We show that all functions which have type $\forall$ (A:Type) ( $\mathrm{x} y: \mathrm{A}) . \mathrm{x}=\mathrm{y} \rightarrow \mathrm{x}=\mathrm{y}$ are identity functions. Let IDp be this type. First we compute the translation of IDp using TranslateRec.

Run TemplateProgram (table $\leftarrow$ TranslateRec emptyTC "IDp" ; ;

tmDefinition "table" table).

The second line defines table as the new translation context, so that we can reuse it later. Then we show that every parametric function of type IDp is pointwise equal to the identity by using the predicate fun $y \Rightarrow x=y$.

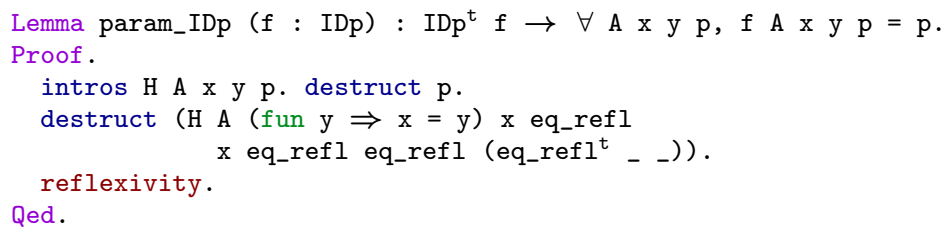

Let's define a function myf $:=p \mapsto p \cdot p^{-1} \cdot p$ and get its parametricity proof using the plugin.

Definition myf: IDp := fun $A x$ y $p \Rightarrow$ eq_trans (eq_trans $p$ (eq_sym $p$ )) $p$. Run TemplateProgram (TranslateRec table "myf").

We reuse here table in which the translation of equality has been recorded. It is then possible to deduce automatically that $p \cdot p^{-1} \cdot p=p$ for all $p$ :

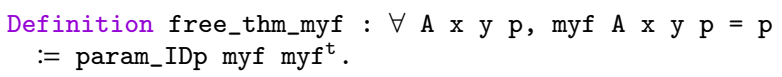




\subsubsection{Times bool translation}

We describe here the use of the plugin with the times bool translation. This translation is a model of $\mathrm{COQ}^{5}$ which negates function extensionality. It will give an example of the use of the command Implement. This example can be found in translations/ times_bool_fun.v.

The translation is defined as follows on variables and dependent products (see Boulier et al. (2017) for a more complete description):

$$
\begin{array}{rlrl}
{[x]_{f}} & :=x & {[\lambda x: A \cdot M]_{f}} & :=\left(\lambda x:[A]_{f} \cdot[M]_{f}, \text { true }\right) \\
{[M N]_{f}} & :=\pi_{1}\left([M]_{f}\right)[N]_{f} & {[\forall x: A \cdot B]_{f}:=\left(\forall x:[A]_{f} \cdot[B]_{f}\right) \times \mathbb{B}}
\end{array}
$$

For this translation, terms and types are translated the same way, hence $\llbracket-\rrbracket_{f}=\left[{ }_{-}\right]_{f}$.

Even if the translation is very simple, this time, going from the ideal world of calculus of constructionsto the real world of CoQ is not as simple as for parametricity. Indeed, when written in CoQ, the translation is no longer fully syntax directed. In CoQ, pairs $(M, N)$ are typed, $M$ and $N$ are not the only arguments, their types are also required:

pair : $\forall$ (A B : Type), $\mathrm{A} \rightarrow \mathrm{B} \rightarrow \mathrm{A} \times \mathrm{B}$

Hence, in the case of lambdas in the definition of the translation, those types have to be provided:

$[f u n(x: A) \Rightarrow t]:=\operatorname{pair}(\forall x:[A] . ? T)$ bool (fun $(x:[A]) \Rightarrow[t])$ true

true is always of type bool, but for the left hand side term, we cannot recover the type ?T from the source term. There is thus a mismatch between the lambdas which are not fully annotated and the pairs which are. There is a similar issue with applications and projections, but this one can be circumvented using primitive projections which are untyped.

A solution is to use the type inference algorithm of Section 2.7 to recover the missing information.

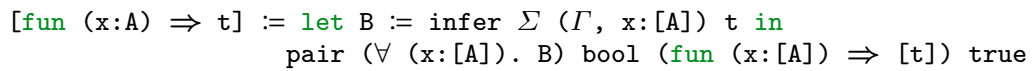

Here we need to have kept track of the global context $\Sigma$ and of the local context $\Gamma$.

The translation function $[-]_{f}$ is thus implemented by:

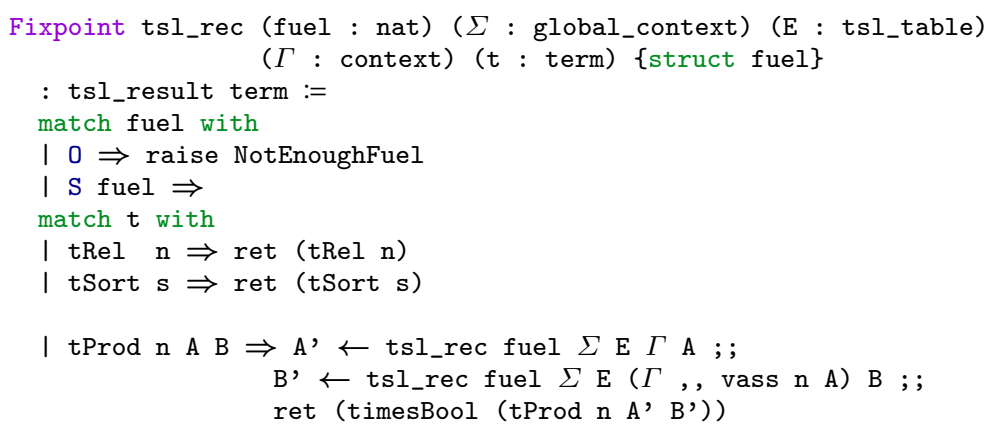

5 In fact, this translation is not completely a model of CoQ: CoQ features $\eta$-conversion on functions, which is incompatible with this translation. 


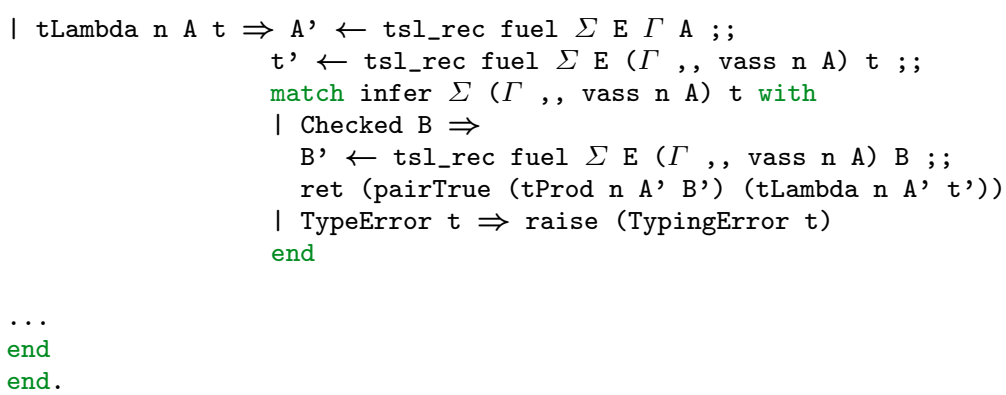

We use a fuel argument because of the non-structural recursive call on $\mathrm{B}$ in the case of lambdas.

We also implemented the translation of some inductive types. For instance, the translation of the inductive foo generates the new inductive foo ${ }^{t}$ :

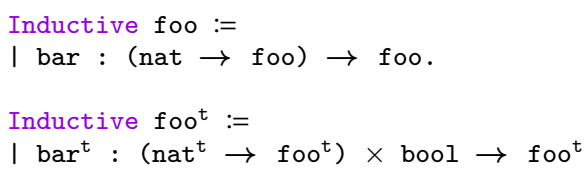

and the translation is extended by:

[foo $]=f \circ o^{t}$

$[$ bar $]=\left(\right.$ bart $^{t} ;$ true)

Example. Let's demonstrate how to use the plugin to negate function extensionality. The type of the axiom we will add to our theory is:

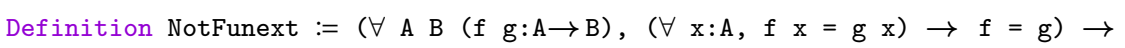

We use TranslateRec to get the translation of eq and False and then we use Implement to inhabit the translation of the NotFunext:

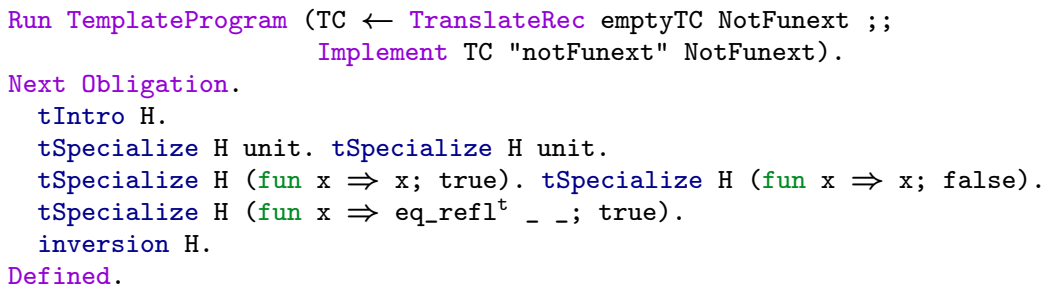

The Implement command generates an obligation whose type is the translation of NotFunext, that is:

$((\forall \mathrm{A},(\forall \mathrm{B}, \quad(\forall \mathrm{f}:(\mathrm{A} \rightarrow \mathrm{B}) \times$ bool, $(\forall \mathrm{g}:(\mathrm{A} \rightarrow \mathrm{B}) \times$ bool,

$\left(\left(\forall \mathrm{x}: \mathrm{A}, \mathrm{eq}^{\mathrm{t}} \mathrm{B}(\pi 1 \mathrm{f} \mathrm{x})(\pi 1 \mathrm{gx})\right) \times\right.$ bool $\rightarrow \mathrm{eq}^{\mathrm{t}}((\mathrm{A} \rightarrow \mathrm{B}) \times$ bool $\left.) \mathrm{f} \mathrm{g}\right)$

$\times$ bool $) \times$ bool $) \times$ bool $) \times$ bool $) \times$ bool $\rightarrow$ False $\left.^{t}\right) \times$ bool

There are a lot of " $\times$ bool", that's why it is convenient that this type is automatically computed. We fill the obligation with the tactics tIntro and tSpecialize which are variants of intro and specialize dealing with the boolean: 


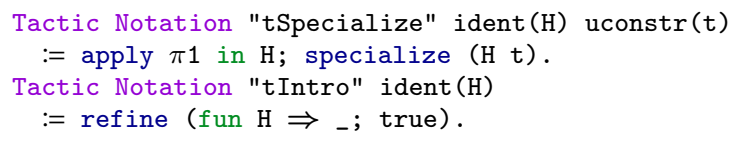

After the obligation is closed (and not before), an axiom notFunext of type NotFunext is declared in the current environment, as it would have been done by:

Axiom notFunext : NotFunext.

A constant notFunext ${ }^{t}$ whose body is the term provided in the obligation is also declared and the mapping (notFunext, notFunext ${ }^{t}$ ) is added in the translation table.

If the translation is correct, the consistency of CoQ is preserved by the addition of this axiom. Let's insist on the fact that it is not fully the case because CoQ has $\eta$-conversion, which is incompatible with this translation.

\subsection{Extraction to $\lambda$-calculus}

As a last example, we show how Template-Coq can be used to extract Coq functions to the weak-call-by-value $\lambda$-calculus (Forster and Kunze, 2019). It is folklore that every function definable in constructive type theory is computable in the classical sense, i.e. in a model of computation. While this statement can not be proven as a theorem inside the type theory of CoQ, similar to parametricity, it is possible to give a computability proof in CoQ for every concrete defined function. The translation from CoQ functions to terms of the $\lambda$-calculus is essentially the identity, since the syntax of CoQ can be seen as a feature-rich, type-decorated $\lambda$-calculus. Special care only has to be taken for fixed-points and inductive types (we do not cover co-inductives).

As concrete target language we use the (weak) call-by-value $\lambda$-calculus as used by Forster and Smolka (2017). The syntax is defined using de Bruijn indices:

$$
s, t, u, v: \text { lterm }::=n|s t| \lambda s \quad(n: \text { nat })
$$

We follow their approach in employing Scott's encoding (Mogensen, 1992; Jansen, 2013) to incorporate inductive types and a fixed-point combinator $\rho$ for recursion.

For instance, the Scott encoding of booleans is defined as $\varepsilon_{\text {bool }}$ true $=\lambda x y . x$ and $\varepsilon_{\text {bool }}$ false $=\lambda x y . y$, or $\lambda \lambda 1$ and $\lambda \lambda 0$ using de Bruijn indices, which we will avoid for examples. For natural numbers, the encodings are $\varepsilon_{\text {nat }} 0=\lambda z s . z$ and $\varepsilon_{\text {nat }}(S n)=$ $\lambda z s . s\left(\varepsilon_{\text {nat }} n\right)$. Note that Scott encodings allow very direct encodings of matches: The COQ term fun $\mathrm{n}$ : nat $\Rightarrow$ match $\mathrm{n}$ with $0 \Rightarrow \ldots$ । $\mathrm{s} \mathrm{n}^{\prime} \Rightarrow \ldots$ end can be directly translated to $\lambda n . n(\ldots)\left(\lambda n^{\prime} \ldots\right)$. We provide a command tmEncode which generates the Scott encoding function for an inductive datatype automatically. We restrict the generation to simple inductive types of the form

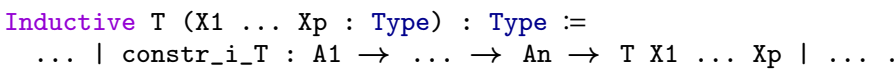

where $\mathrm{Aj}$ for $1 \leq \mathrm{j} \leq \mathrm{n}$ is either encodable or exactly $\mathrm{T} \mathrm{X1} \ldots \mathrm{Xn}$. For such a fully instantiated inductive type $\mathrm{B}=\mathrm{T} \mathrm{X} 1 \ldots \mathrm{Xp}$ with $\mathrm{n}$ constructors we define the encoding function $\varepsilon_{\mathrm{B}}$ as follows:

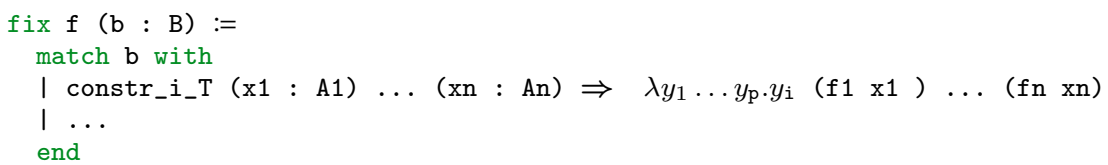


where $\mathrm{fj}$ for $1 \leq \mathrm{j} \leq \mathrm{n}$ is a recursive call $\mathrm{f}$ if $\mathrm{Aj}=\mathrm{B}$, or $\varepsilon_{\mathrm{Aj}}$ otherwise. To be able to obtain the encoding function $\varepsilon_{\mathrm{Aj}}$, we could use translation tables as before. Instead, we demonstrate an alternative way using a type class of encodable types defined as follows:

Class encodable (A : Type) := enc_f : A $\rightarrow$ lterm.

Then, to generate, for instance, the Scott encoding of the type 1term itself, one first has to generate the Scott encoding for natural numbers:

Run TemplateProgram (tmEncode "nat_enc" nat).

Run TemplateProgram (tmEncode "lterm_enc" lterm).

This will define nat_enc : encodable nat and lterm_enc : encodable lterm. The second command uses the tmInferInstance operation of the TemplateMonad to find the instance of encodable nat defined before. If no instance is found, an obligation of type encodable nat is opened.

To extract functions, we proceed similarly. We restrict the extraction to a simple polymorphic subset of CoQ without dependent types. We call a type $A$ admissible if $A$ is of the form $\forall X_{1} \ldots X_{n}$ : Type. $B_{1} \rightarrow \cdots \rightarrow B_{m}$ with $B_{m} \neq$ Type. Terms $a: A$ are admissible if $A$ is admissible and if all constants $c: C$ that are proper subterms of $a$ are either (a) admissible and occur syntactically on the left hand side of an application fully instantiating the type-parameters of $c$ with constants or (b) of type Type and occur syntactically on the right hand side of an application instantiating type parameters. For instance, the definition of the function @map A B : list A $\rightarrow$ list B is admissible:

Definition map (A B : Type) $:(A \rightarrow B) \rightarrow$ list $A \rightarrow$ list $B:=$ fun $f \Rightarrow$

fix map $:=$ match 1 with $\mid[] \Rightarrow$ @nil B | a : : $t \Rightarrow$ @cons B (f a) (map 1) end.

We again define a type class to look up previously extracted terms:

Class extracted $\{\mathrm{A}:$ Type $\}$ (a : A) := int_ext : lterm.

For constants (and constructors) occurring as subterms the tmInferInstance operation is used again to obtain the respective extractions. We define commands tmExtract and tmExtractConstr which can be used to extract functions and constructors. To extract the full polymorphic map function, we use CoQ's section mechanism:

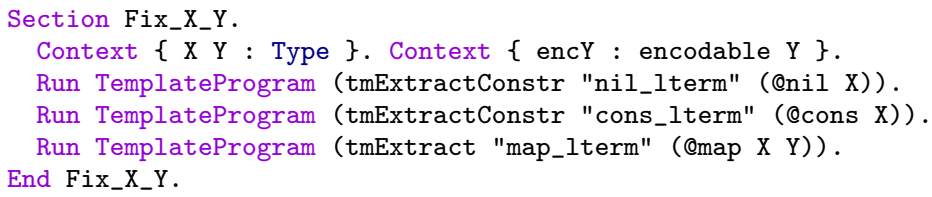

This will define map_lterm : $\forall \mathrm{X} Y\{\mathrm{H}:$ encodable $\mathrm{Y}\}$, extracted (@map X Y) and register it as an instance of the type class extracted.

To prove that the extracted terms are indeed correct, we provide a logical relation $t_{a} \sim a$ read as $t_{a}$ computes $a$ and a set of Ltac tactics which will automatically establish this relation. We wrap extracted terms together with the relation into a type class computable. We use MetaCoQ's ability to run monadic operations inside tactics to implement a tactic extract which uses tmExtract and the Ltac tactics to allow for automatic computability proofs. Since this is not directly related to METACoQ, we omit the details here and refer to Forster and Kunze (2019).

To automatically verify terms, we again use tmInferInstance to obtain the correctness proofs for previously extracted constants or constructors. The correctness lemma for fix w.r.t weak call-by-value reduction $\succ$ can be stated in general as $\rho u v \succ^{*} u(\rho u) v$ 
for closed abstractions $u, v$. For match, the correctness lemmas depend on the type of the discriminee and we provide an operation tmGenEncode generating both the encoding function and the correctness lemma for the corresponding match.

For instance, in order to prove the computability of addition, a user has to generate the encoding of natural numbers and extract the successor function first:

Run TemplateProgram (tmGenEncode "nat_enc" nat).

Hint Resolve nat_enc_correct : Lrewrite.

Instance lterm_S : computable S.

Proof. extract constructor. Qed.

Instance lterm_add : computable add.

Proof. extract. Qed.

\section{Running plugins natively in OCaml}

The approach of writing CoQ plugins in CoQ, as illustrated above, has several advantages. First, functions written in COQ are amenable to verification, and second, plugins can be written and iterated on quickly within a CoQ buffer. However, one major disadvantage is that, CoQ programs can not leverage efficient representations, algorithms, and compilers available for other languages, which makes CoQ programs comparatively slow. This is especially a problem for our plugins which process the raw syntax of terms (Ast.term) which can be very verbose.

To mitigate the performance problem, it is common practice to run verified CoQ programs after extraction to OCAML. Extraction gives us access, to both the efficiency of native code, and provides a declarative way to replace inefficient CoQ types with efficient, machine-optimized types and operations in OCAML. During extraction, the CoQ type term is extracted to an OCAML datatype, say coq_term_ext, and programs operate on that representation. To interface these computations with the CoQ internals, which is necessary for plugins, we implemented functions that convert CoQ's kernel representation of terms, i.e. constr, to coq_term_ext. Just the translation in this one direction provides sufficient functionality to implement plugins such as the CERTICoQ compiler which translates CoQ terms into CompCert's Clight intermediate language. More sophisticated plugins, such as the parametricity plugin, need to use both reification and reflection in a dynamic way. This poses the challenge of providing and implementation of TemplateMonad in OCAML so that it can be run after extraction.

Unfortunately, the use of meta-language Coq terms to represent Coq terms in the template monad, as opposed to abstract syntax terms, makes extracting TemplateMonad programs impossible. For example, consider the type of tmPrint, $\forall$ A, A $\rightarrow$ TemplateMonad unit. Under extraction, the value of type A will be extracted to an OCAML value of the extracted type corresponding to A. This does not match the intended semantics of the template monad, however, because we wish to print the CoQ term.

To address this problem, we define an extractable variant of the TemplateMonad which we call TM for the purposes of this presentation. Rather than using the (inlined) type $\{t$ :Type \& $t\}$ to represent CoQ terms, it instead uses the Ast.term type. Figure 4 shows the constructors that changed between TM and TemplateMonad. In addition to the modified constructors, TM drops tmQuote, tmQuoteRec, tmUnqote, and tmUnquoteTyped, none of which make sense with the new representation of terms. The unquote commands 


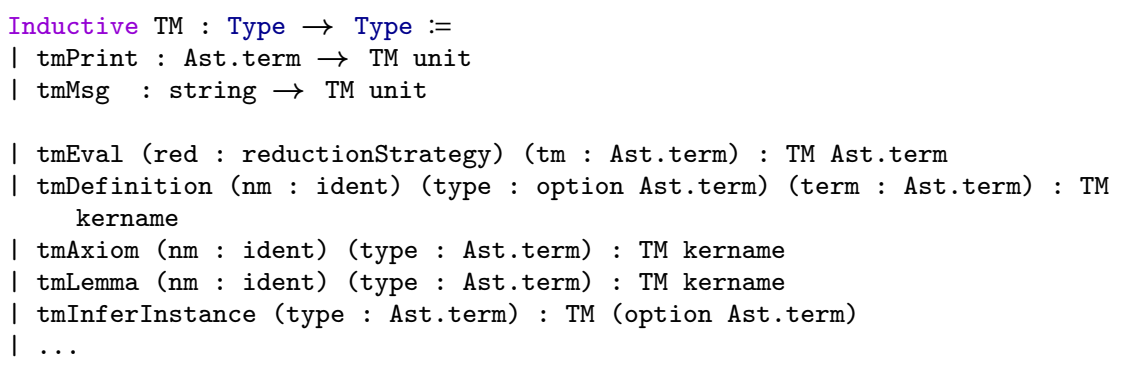

Fig. 4 Modified constructors in TM and TemplateMonad.

could, theoretically, be implemented by an interpreter, but the interface between this interpreter and the native OCAML program would not be sensible.

As a by-product of the phase separation, we solve an additional problem that is necessary. The TemplateMonad type lives in Prop in order to get impredicativity and avoid problems when manipulating terms of higher universes. Concretely, note that we can not existentially quantify over universe levels, which one might have to do if unquoting a term of type TemplateMonad. While we have not needed to unquote TemplateMonad values, in practice, we have experienced universe issues with an earlier version of TemplateMonad which was defined in Type.

Using the Phase Split Monad The phase split comes at the cost of some convenience. In the original TemplateMonad, we could write, tmDefinition "one" 1 . In the phase split monad, we must construct the term representation of 1 explicitly. To ease this, we define a the $<\% \mathrm{t} \%>$ notation, inspired by MetaOCaml's $.<\mathrm{t}>$., which desugars to the quoted version of $t$ using CoQ's tactics-in-terms feature. Using this feature, we can adapt the simple declaration above as tmDefinition "one" $\langle \% 1 \%\rangle$.

Things become slightly more complicated when the term to quote is built dynamically, for example: fun $\mathrm{x} y$ : Ast.term $\Rightarrow$ tmDefinition "add_them" $<\% \mathrm{x}+\mathrm{y} \%>$. Currently, to achieve this, we must build the syntax directly: tApp $<\%$ plus $\%>$ ( $x::$ y : : nil). Proper multi-stage languages, such as MetaOCaml, address this through a splicing operator where the above could be written . $<\sim x+. \sim y>$.. We leave implementing improved splicing to future work.

Limitations of the Phase Split Monad While the programs can be slightly more verbose, from a practical point of view, the phase split does not decrease the expressivity of the monad ${ }^{6}$. To see this, consider an arbitrary program that uses tmQuote. The value to quote is either a literal in the program (in which case we can simply use $\langle \% \%>$ ), comes from the caller (in which case we ask the caller to pass the quoted value instead), or is a hybrid of the two (see above)

In general, we found that, in most instances, adapating code simply required phasesplitting the top-level function. For example, a template program that might previously have taken an arbitrary value now takes a term, and the caller of the function performs the quoting on their side. Readers familiar with TemplateHaskell Sheard and Jones (2002b) will note that this style is also employed there.

\footnotetext{
6 One exception is with tmQuoteRec which requires recursion which can not be proved structurally recursive to implement.
} 
Performance Our largest use case for running plugins after extraction is lens ${ }^{7}$ generation for Coq records. This plugin takes the fully qualified name of a record in the environment and defines a lens for each field of the record. A lens for a field of record can be used to project that field or update that field (while keeping the other fields constant). The plugin's implementation invokes the tmQuoteInductive to get the definition of the record, computes the body and the type of the lens for each field, and then defines each of those lenses by using the tmMkDefinition command. Although in our verification work, we typically have records of only a few fields, to very roughly estimate the execution-time savings in general, we tested the lens plugin both with and without extraction on a record with 30 fields. The execution time was respectively 0.774 second and 0.047 second: the extracted version ran at least 10 times faster. We observed more speedups on records with more fields.

\section{Related Work and Future Work}

Meta-Programming is a whole field of research in the programming languages community, we will not attempt to give a detailed review of related work here. In contrast to most work on meta-programming, we provide a very rough interface to the object language: one can easily build ill-scoped and ill-typed terms in our framework, and staging is basic. However, with typing derivations we provide a way to verify meta-programs and ensure that they do make sense.

The closest cousin of our work is the Typed Syntactic Meta-Programming (Devriese and Piessens, 2013) proposal in AGDA, which provides a well-scoped and well-typed interface to a denotation function, that can be used to implement tactics by reflection. We could also implement such an interface, asking for a proof of well-typedness on top of the tmUnquoteTyped primitive of our monad.

Intrinsically typed representations of terms in dependent type-theory is an area of active research. Most solutions are based on extensions of Martin-Löf Intensional Type Theory with inductive-recursive or quotient inductive-inductive types (Chapman, 2009; Altenkirch and Kaposi, 2016), therefore extending the meta-theory. Recent work on verifying soundness and completeness of the conversion algorithm of a dependent type theory (with natural numbers, dependent products and a universe) in a type theory with IR types (Abel et al., 2018) gives us hope that this path can nonetheless be taken to provide the strongest guarantees on our conversion algorithm. The intrinsically-typed syntax used there is quite close to our typing derivations.

Another direction is taken by the (Euf certified compiler (Mullen et al., 2018), which restricts itself to a fragment of CoQ for which a total denotation function can be defined, in the tradition of definitional interpreters advocated by Chlipala (2011). This setup should be readily accomodated by TEMPLATE-COQ.

The translation + plugin technique paves the way for certified translations and the last piece will be to prove correctness of such translations. By correctness we mean computational soundness and typing soundness (see Boulier et al. (2017)), and both can be stated in Template-Coq. Anand has made substantial attempts in this direction to prove, in TEMPlate-CoQ, the computational soundness of a variant of parametricity providing stronger theorems for free on propositions (Anand and Morrisett, 2018). This included as a first step a move to named syntax that could be reused in other

\footnotetext{
7 this is inspired by lenses in Haskell: http://lens.github.io
} 
translations. Our long term goal is to leverage the translation + plugin technique to extend the logical and computational power of CoQ using, for instance, the forcing translation (Jaber et al., 2016) or the weaning translation (Pédrot and Tabareau, 2017).

The last direction of extension is to build higher-level tools on top of the syntax: the unification algorithm described in (Ziliani and Sozeau, 2017) is our first candidate. Once unification is implemented, we can look at even higher-level tools: elaboration from concrete syntax trees, unification hints like canonical structures and type class resolution, domain-specific and general purpose tactic languages. A key inspiration in this regard is the work of Malecha and Bengtson (2016) which implemented this idea on a restricted fragment of CIC.

\section{Acknowledgments}

This work is supported by the CoqHoTT ERC Grant 64399 and the NSF grants CCF-1407794, CCF-1521602, and CCF-1646417.

\section{References}

Abel A, Öhman J, Vezzosi A (2018) Decidability of conversion for type theory in type theory. PACMPL 2(POPL):23:1-23:29, DOI 10.1145/3158111, URL http://doi . acm. org/10.1145/3158111

Altenkirch T, Kaposi A (2016) Type theory in type theory using quotient inductive types. ACM, New York, NY, USA, POPL '16, pp 18-29, DOI 10.1145/2837614.2837638, URL http://doi.acm.org/10.1145/2837614.2837638

Anand A, Morrisett G (2018) Revisiting Parametricity: Inductives and Uniformity of Propositions. In: CoqPL'18, Los Angeles, CA, USA

Anand A, Appel A, Morrisett G, Paraskevopoulou Z, Pollack R, Belanger OS, Sozeau M, Weaver M (2017) CertiCoq: A verified compiler for Coq. In: CoqPL, Paris, France, URL http://conf.researchr.org/event/CoqPL-2017/ main-certicoq-a-verified-compiler-for-coq

Armand M, Grégoire B, Spiwack A, Théry L (2010) Extending Coq with Imperative Features and Its Application to SAT Verification. In: Kaufmann M, Paulson LC (eds) Interactive Theorem Proving, Springer, pp 83-98

Barras B (1999) Auto-validation d'un système de preuves avec familles inductives. Thèse de doctorat, Université Paris 7, URL http://pauillac.inria.fr/ barras/publi/ these_barras.ps.gz

Bernardy JP, Jansson P, Paterson R (2012) Proofs for free: Parametricity for dependent types. Journal of Functional Programming 22(2):107-152

Boulier S, Pédrot PM, Tabareau N (2017) The next 700 syntactical models of type theory. In: CPP'17, Paris, France, ACM, pp 182-194

Chapman J (2009) Type Theory Should Eat Itself. Electronic Notes in Theoretical Computer Science 228:21 - 36, DOI https://doi.org/10.1016/j.entcs.2008.12.114, URL http://www.sciencedirect.com/science/article/pii/S157106610800577X, proceedings of LFMTP 2008

Chlipala A (2011) Certified Programming with Dependent Types. MIT Press

Cormen TH, Leiserson CE, Rivest RL, Stein C (2009) Introduction to algorithms. MIT press 
Devriese D, Piessens F (2013) Typed syntactic meta-programming. In: Proceedings of the 18th ACM SIGPLAN International Conference on Functional Programming, ACM, ICFP '13, URL http://doi.acm.org/10.1145/2500365.2500575

Ebner G, Ullrich S, Roesch J, Avigad J, de Moura L (2017) A Metaprogramming Framework for Formal Verification. In: Proceedings of the 22st ACM SIGPLAN Conference on Functional Programming (ICFP 2017), ACM Press, Oxford, UK, pp $34: 1-34: 29$

Forster Y, Kunze F (2016) Verified Extraction from Coq to a Lambda-Calculus. In: Coq Workshop 2016, URL https://www.ps.uni-saarland.de/ ${ }^{\text {forster/ }}$ coq-workshop-16/abstract-coq-ws-16.pdf

Forster Y, Kunze F (2019) A certifying extraction with time bounds from coq to call-byvalue $\lambda$-calculus. CoRR abs/1904.11818, URL http://arxiv.org/abs/1904.11818, 1904.11818

Forster Y, Smolka G (2017) Weak call-by-value lambda calculus as a model of computation in Coq. In: ITP 2017, Springer, pp 189-206

Herbelin H, Spiwack A (2013) The rooster and the syntactic bracket. In: Matthes R, Schubert A (eds) 19th International Conference on Types for Proofs and Programs, TYPES 2013, April 22-26, 2013, Toulouse, France, Schloss Dagstuhl - Leibniz-Zentrum fuer Informatik, LIPIcs, vol 26, pp 169-187, DOI 10.4230/LIPIcs.TYPES.2013.169, URL https://doi.org/10.4230/LIPIcs.TYPES.2013.169

Jaber G, Lewertowski G, Pédrot PM, Sozeau M, Tabareau N (2016) The definitional side of the forcing. In: LICS'16, New York, NY, USA, pp 367-376, DOI 10.1145/ 2933575.2935320, URL http://doi.acm.org/10.1145/2933575.2935320

Jansen JM (2013) Programming in the $\lambda$-calculus: From Church to Scott and back. In: The Beauty of Functional Code, LNCS, vol 8106, Springer, pp 168-180

Keller C, Lasson M (2012) Parametricity in an impredicative sort. CoRR abs/1209.6336, URL http://arxiv.org/abs/1209.6336, 1209.6336

Lasson M (2014) Canonicity of Weak $\omega$-groupoid Laws Using Parametricity Theory. In: Proceedings of the 30th Conference on the Mathematical Foundations of Programming Semantics (MFPS XXX), DOI 10.1016/j.entcs.2014.10.013

Malecha G, Bengtson J (2016) Extensible and efficient automation through reflective tactics. In: ESOP 2016, DOI 10.1007/978-3-662-49498-1_21, URL http://dx.doi. org/10.1007/978-3-662-49498-1_21

Malecha GM (2014) Extensible proof engineering in intensional type theory. PhD thesis, Harvard University, URL http://gmalecha.github.io/publication/2015/02/01/ extensible-proof-engineering-in-intensional-type-theory.html

Mogensen TÆ (1992) Efficient self-interpretations in lambda calculus. J Funct Program 2(3):345-363

Mullen E, Pernsteiner S, Wilcox JR, Tatlock Z, Grossman D (2018) Cuf: minimizing the coq extraction TCB. In: Proceedings of CPP 2018, pp 172-185, DOI 10.1145/3167089, URL http://doi.acm.org/10.1145/3167089

Pédrot P, Tabareau N (2017) An effectful way to eliminate addiction to dependence. In: LICS'17, Reykjavik, Iceland, pp 1-12, DOI 10.1109/LICS.2017.8005113, URL https://doi.org/10.1109/LICS. 2017.8005113

Reynolds JC (1983) Types, abstraction and parametric polymorphism. In: IFIP Congress, pp 513-523

Sheard T, Jones SP (2002a) Template meta-programming for haskell. SIGPLAN Not 37(12):60-75, DOI 10.1145/636517.636528, URL http://doi.acm.org/10.1145/ 636517.636528 
Sheard T, Jones SP (2002b) Template meta-programming for haskell. In: Proceedings of the 2002 ACM SIGPLAN Workshop on Haskell, ACM, New York, NY, USA, Haskell '02, pp 1-16, DOI 10.1145/581690.581691, URL http://doi.acm.org/10. $1145 / 581690.581691$

Sozeau M (2007) Program-ing Finger Trees in Coq. ACM, New York, NY, USA, ICFP '07, pp 13-24, DOI 10.1145/1291151.1291156, URL http://doi.acm.org/10.1145/ 1291151.1291156

Taha W, Sheard T (1997) Multi-stage programming with explicit annotations. ACM, New York, NY, USA, PEPM '97, pp 203-217, DOI 10.1145/258993.259019, URL http://doi.acm.org/10.1145/258993.259019

Wadler P (1989) Theorems for free! In: Functional Programming Languages and Computer Architecture, ACM Press, pp 347-359

Van der Walt P, Swierstra W (2013) Engineering Proof by Reflection in Agda. In: Implementation and Application of Functional Languages, Springer

Ziliani B, Sozeau M (2017) A Comprehensible Guide to a New Unifier for CIC Including Universe Polymorphism and Overloading. Journal of Functional Programming 27:e10, DOI 10.1017/S0956796817000028, URL http://www.irif .univ-paris-diderot.fr/ sozeau/research/publications/drafts/unification-jfp.pdf

Ziliani B, Dreyer D, Krishnaswami NR, Nanevski A, Vafeiadis V (2015) Mtac: A Monad for Typed Tactic Programming in Coq. Journal of Functional Programming 25, DOI 10.1017/S0956796815000118, URL https://doi.org/10.1017/S0956796815000118 